\title{
Biophysical and biological contributions of polyamine-coated carbon nanotubes and bidimensional buckypapers in the delivery of miRNAs to human cells
}

This article was published in the following Dove Press journal:

International Journal of Nanomedicine

\author{
Antonella Celluzzi ${ }^{1, *}$ \\ Alessandro Paolini',* \\ Valentina D'Oria' \\ Roberta Risoluti ${ }^{2}$ \\ Stefano Materazzi ${ }^{2}$ \\ Marco Pezzullo' \\ Stefano Casciardi ${ }^{3}$ \\ Simona Sennato ${ }^{4}$ \\ Federico Bordi ${ }^{4}$ \\ Andrea Masotti ${ }^{1}$
}

'Bambino Gesù Children's Hospital, IRCCS, Research Laboratories,

${ }^{2}$ Department of Chemistry, Sapienza University of Rome, ${ }^{3}$ Department of Occupational and Environmental Medicine, Epidemiology and Hygiene, National Institution for Insurance Against Accidents at Work (INAIL Research), Monte Porzio Catone, ${ }^{4}$ CNR-ISC UOS Roma, Department of Physics, Sapienza University of Rome, Roma, Italy

*These authors contributed equally to this work
Correspondence: Andrea Masotti Bambino Gesù Children's Hospital, IRCCS, Research Laboratories, V.le di San Paolo I5, 00146 Rome, Italy

Tel +3906 68592650

Fax +3906 68592904

Email andrea.masotti@opbg.net

\begin{abstract}
Recent findings in nanomedicine have revealed that carbon nanotubes (CNTs) can be used as potential drug carriers, therapeutic agents and diagnostics tools. Moreover, due to their ability to cross cellular membranes, their nanosize dimension, high surface area and relatively good biocompatibility, CNTs have also been employed as a novel gene delivery vector system. In our previous work, we functionalized CNTs with two polyamine polymers, polyethyleneimine (PEI) and polyamidoamine dendrimer (PAMAM). These compounds have low cytotoxicity, ability to conjugate microRNAs (such as miR-503) and, at the same time, transfect efficiently endothelial cells. The parameters contributing to the good efficiency of transfection that we observed were not investigated in detail. In fact, the diameter and length of CNTs are important parameters to be taken into account when evaluating the effects on drug delivery efficiency. In order to investigate the biophysical and biological contributions of polymer-coated CNTs in delivery of miRNAs to human cells, we decided to investigate three different preparations, characterized by different dimensions and aspect ratios. In particular, we took into account very small CNTs, a suspension of CNTs starting from the commercial product and a 2D material based on CNTs (ie, buckypapers [BPs]) to examine the transfection efficiency of a rigid scaffold. In conclusion, we extensively investigated the biophysical and biological contributions of polyamine-coated CNTs and bidimensional BPs in the delivery of miRNAs to human cells, in order to optimize the transfection efficiency of these compounds to be employed as efficient drug delivery vectors in biomedical applications.
\end{abstract}

Keywords: carbon nanotubes, buckypapers, polyamines, cytotoxicity, cell transfection

\section{Introduction}

In the last few years, many studies about the use of carbon nanotubes (CNTs) in several research areas (ie, nanotechnology, physics, chemistry, tissue engineering, biology and biomedicine) outlined the importance of this kind of nanomaterial. ${ }^{1-4}$ Recent findings in nanomedicine have revealed that CNTs can be used as potential drug carriers, therapeutic agents and diagnostics tools as well..$^{5-10}$ In particular, due to their ability to cross cellular membranes, their nanosize dimension, high surface area and relatively good biocompatibility, CNTs have also been employed as a novel gene delivery vector system. ${ }^{11-13}$ However, one of the major drawbacks is their low solubility and high propensity to aggregate in aqueous solutions. To avoid aggregation of unmodified (or "pristine") CNTs, mainly due to their hydrophobicity, a proper surface functionalization is required to suspend CNTs and impart them a good biocompatibility. ${ }^{14}$ submit your manuscript Dovepress fy in 0
International Journal of Nanomedicine 2018:13 1-18

(c) (7) (5) 2018 Celluzzi et al. This work is published and licensed by Dove Medical Press Limited. The full terms of this license are available at https://www.dovepress.com/terms.php (c) hereby accept the Terms. Non-commercial uses of the work are permitted without any further permission from Dove Medical Press Limited, provided the work is properly attributed. For permission for commercial use of this work, please see paragraphs 4.2 and 5 of our Terms (https://www.dovepress.com/terms.php). 
Many different approaches have been adopted to functionalize CNTs (ie, oxidation, covalent and noncovalent functionalization, etc), but the preferred way of functionalization for medical applications is functionalization with proteins due to their excellent biocompatibility. ${ }^{15}$ In many cases, noncovalent functionalization methods are preferable since molecules can be attached to nanotubes straightforwardly and without affecting other molecular interactions. ${ }^{16}$ In particular, the noncovalent derivatization exploits the hydrophobic nature of CNTs (especially $\pi-\pi$ interactions and Van der Waals' forces) to coat them with amphiphilic molecules such as poly(ethylene glycol) (PEG), polyethyleneimine (PEI) or polyamidoamine dendrimer (PAMAM).

These polymers are able to impart hydrophilicity to CNTs, which facilitates their suspension in aqueous solutions. Moreover, absorbed polymers generate a positively charged surface able to bind negatively charged nucleic acids (ie, siRNAs or plasmid DNA) through electrostatic interactions. These properties allow polymer-coated CNTs to be efficient transfection agents. ${ }^{8,17-20}$

In our previous work, we functionalized CNTs with two polyamine polymers, PEI and PAMAM. These compounds have low cytotoxicity, ability to conjugate microRNAs (such as miR-503) and, at the same time, transfect efficiently endothelial cells. ${ }^{21}$ However, the parameters contributing to the good efficiency of transfection that we observed were not investigated in detail. In fact, the diameter and length of CNTs are important parameters to be taken into account when evaluating the effects on drug delivery efficiency. ${ }^{22}$ Many studies have focused the attention on this aspect, but authors observed that the transfection efficiency is inversely correlated with the length of CNTs. ${ }^{23-25}$ Therefore, in order to find the optimal CNT-based drug delivery system coated with polyamine polymers for delivery of miRNAs to human cells, we decided to investigate three different preparations, characterized by different dimensions and aspect ratios. In particular, we took into account very small CNTs, a suspension of CNTs starting from the commercial product and a 2D material based on CNTs (ie, buckypapers [BPs]) to examine the transfection efficiency of a rigid scaffold. ${ }^{26} \mathrm{BPs}$ are randomly entangled and densely packed CNT networks, which have been employed in retina and iris pigment epithelial transplantation ${ }^{27}$ as actuators, ${ }^{28}$ biosensors,${ }^{29}$ carriers for drug delivery $^{30}$ and finally as scaffolds for tissue engineering. ${ }^{31}$ Although few studies have characterized the properties of BPs on cell adhesion, cell response and cytotoxicity ${ }^{32-34}$ and proposed the use of BPs in medical applications, ${ }^{35-37}$ the use of BPs as transfecting agents for nucleic acids has never been proposed. Therefore, our systematic investigation of the biophysical and biological properties of bidimensional CNT-based drug delivery systems is not only original and innovative but also very useful for a further extension of this work aimed at employing BPs as scaffolds or supports for cell growth.

In conclusion, in this study we extensively investigated the biophysical and biological contributions of polyaminecoated CNTs and bidimensional BPs in the delivery of miRNAs to human cells, to optimize the transfection efficiency of these compounds to be employed as efficient drug delivery vectors in biomedical applications.

\section{Materials and methods Materials}

Multi-walled CNTs were purchased from Cheap Tubes Inc (Cambridgeport, MA, USA). PEI polymer (cat no 408727), PAMAM generation 5 (PAMAM G=5, cat no 536709), thiazolyl blue tetrazolium bromide (MTT, cat no M5655) and a fluorescein (FAM)-labeled oligonucleotide mimicking the precursor form of miRNA-503 (FAM-mir-503, batch no HA08019806) were purchased from Sigma-Aldrich Co. (St Louis, MO, USA) and used as received. Human Embryonic Kidney 293 cells (HEK 293T) were purchased from the American Tissue Culture Collection (ATCC, Manassas, VA, USA).

\section{Preparation of polyamine-coated CNTs}

For coating, CNTs (10 mg) were dispersed in three different solutions (2 mL) of PEI or PAMAM (10\%, 20\% and 40\% $\mathrm{w} / \mathrm{w}$, respectively). The three suspensions were placed in a bath sonicator for $30 \mathrm{~min}$ and further stirred for $72 \mathrm{~h}$ at room temperature. A part of the sample was used "as is" (named PEI-CNTs and PAM-CNTs), while the remaining part was centrifuged at $20,800 \times g$ for $30 \mathrm{~min}$ and the collected supernatant employed for in vitro assays (named supPEI-CNTs and supPAM-CNTs).

\section{Quantification of polymer bound to CNTs}

To assess the amount of polymer (PEI or PAMAM) bound to CNTs in the three solutions tested, we employed thermogravimetric analysis (TGA). TGA curves were recorded using a TGA7 equipment (PerkinElmer Inc., Waltham, MA, USA). The samples investigated were heated in platinum crucibles in the temperature range $20^{\circ} \mathrm{C}-850^{\circ} \mathrm{C}$, in pure nitrogen or air atmosphere (gaseous mixture of nitrogen and oxygen with $80 \%$ and $20 \%, \mathrm{v} / \mathrm{v}$, respectively) under a flow rate of $100 \mathrm{~mL} \mathrm{~min}^{-1}$. The scanning rate for the best resolution was found to be $10^{\circ} \mathrm{C} \mathrm{min}-1$. To ensure an accurate measurement 
of sample temperature, the calibration was performed using the Curie-point transition of standard metals, according to manufacturer's recommendations. Each sample was analyzed in triplicate.

\section{Dynamic light scattering (DLS) and electrophoresis measurements}

NanoZetaSizer (Malvern Instruments, Malvern, UK) apparatus has been employed to determine size and $\zeta$-potential. For size measurements, a backscatter detection was employed. The instrument is equipped with a $5 \mathrm{~mW} \mathrm{He}-\mathrm{Ne}$ laser operating at a wavelength of $633 \mathrm{~nm}$. To get the size distribution of samples, the correlation functions were analyzed as intensity-averaged particle size using the DTS Software (v.7.01) within the framework of the Mie theory by using the non-negative least squares algorithm. ${ }^{38}$ For the measurement of the electrophoretic mobility $\mu$, Laser Doppler velocimetry with Phase Analysis Light Scattering (PALS) implemented with the patented mode M3 (mixed mode measurement) has been employed. ${ }^{39}$ This configuration allowed elimination of electro-osmotic effects and improvement of accuracy and repeatability of measurements. Preliminary to electrophoretic measurements, Zetasizer Nano automatically measures the conductivity of the sample directly in the $\zeta$-potential cell to establish the appropriate voltage to be applied to avoid sample damage and improve measurement quality. The $\zeta$-potential was determined from the measured electrophoretic mobility $\mu$ by using the Smoluchowski approximation: $\zeta=\mu \eta / \varepsilon$, where $\varepsilon$ and $\eta$ are the permittivity and the viscosity, respectively, of the suspending medium. As in other electrophoretic investigations on $\mathrm{CNTs},{ }^{40}$ this approximation has been used even though it is rigorously valid only for spherical particles. The Smoluchowski approximation also assumes that the particle permittivity is much less than $\varepsilon$, which is true for semiconducting CNTs but not for metallic CNTs. Depending on the magnitude of the $\zeta$-potential, the spherical Smoluchowski approximation may overestimate the actual $\zeta$-potential by up to $20 \%$. Both size and electrophoretic mobility measurements have been performed in thermostated cells at $25^{\circ} \mathrm{C}$ and have been carried out in triplicate.

\section{Transmission electron microscopy (TEM)}

Samples for TEM observation were prepared by depositing $20 \mu \mathrm{L}$ of the nanotube suspension on a gold grid (mesh 1,000) without using any supporting amorphous carbon film. Immediately after deposition, the excess of liquid was removed by touching the grid with a piece of filter paper. Measurements were carried out using a FEI TECNAI 12 G2 Twin (FEI Company,
Hillsboro, OR, USA), operating at $120 \mathrm{kV}$ and equipped with an electron energy filter (Gatan image filter) and a slow-scan charge-coupled device camera (Gatan multiscan).

\section{Gel retardation assays}

Agarose gel retardation assays were performed following standard procedures. ${ }^{41}$ Polymer-coated CNTs and FAMmir-503 (50 nM) were mixed at CNT/miRNA weight ratios of 5:1, 10:1 and 20:1 (equivalent to 5,10 and $20 \mu \mathrm{g} / \mathrm{mL}$ of CNTs, respectively). Compounds were incubated at room temperature for $15 \mathrm{~min}$. Then, complexes were loaded into $1 \%$ agarose gel (in tris-acetate-EDTA buffer) and run for $30 \mathrm{~min}$ at $90 \mathrm{~V}$. Hoechst $33258(10 \mathrm{mg} / \mathrm{mL})$ was added to the agarose gel as intercalating dye. Gels were visualized by ultraviolet (UV) and visible light. To assess the stability of CNT/miRNA complexes, heparin 5,000 U/mL (Teva Pharmaceutical Industries, Petah Tikva, Israel) was added to CNT/ miRNA complexes, and the displacement of the complex was monitored after incubating for 30 and $60 \mathrm{~min}$.

\section{MTT assay}

To assess the cytotoxicity of polymer-coated CNTs, Human Embryonic Kidney 293 cells (HEK 293T) were seeded on a 96-well plate at a density of $1 \times 10^{5}$ cells $/ \mathrm{mL}$ per well. After $24 \mathrm{~h}$, cells were treated with PEI (4, 8 and $12 \mu \mathrm{g} / \mathrm{mL})$, with PAMAM $(4,8$ and $20 \mu \mathrm{g} / \mathrm{mL})$ and with PEI-CNTs or PAM-CNTs at concentrations of 10, 20, 30 and $50 \mu \mathrm{g} / \mathrm{mL}$.

To test the supernatant toxicity also, cells were treated with supPEI-CNTs and supPAM-CNTs at the same concentration of polymers $(4,8,12$ and $20 \mu \mathrm{g} / \mathrm{mL})$ or CNTs $(10$, 20,30 and $50 \mu \mathrm{g} / \mathrm{mL}$ ).

Each sample was analyzed in triplicate. After $24 \mathrm{~h}$, the plate was centrifuged at $3,320 \times g$ for $10 \mathrm{~min}$, and MTT solution $(50 \mu \mathrm{L}, 5 \mathrm{mg} / \mathrm{mL})$ was added to each well. After additional $3 \mathrm{~h}$, the medium was removed and the obtained formazan crystals were solubilized in $200 \mu \mathrm{L}$ of pure dimethyl sulfoxide (DMSO). To eliminate the risk of obtaining falsepositive results due to the absorbance of CNTs, the plate was centrifuged at $3,320 \times g$ for $10 \mathrm{~min}$ and $100 \mu \mathrm{L}$ of the formazan crystal solution was transferred in a new 96-well plate according to the protocol described in Worle-Knirsch et al's study. ${ }^{42}$ The absorbance $(570 \mathrm{~nm})$ of formazan crystals was measured by an enzyme-linked immunosorbent assay (ELISA) plate reader (Benchmark Plus; Bio-Rad Laboratories Inc., Hercules, CA, USA). The growth medium was considered as the negative control (100\% cell viability), whereas cells treated with DMSO were considered as the positive control $(0 \%$ cell viability). 


\section{Cellular uptake of miRNAs complexed to polyamine-coated CNTs}

Human Embryonic Kidney 293 cells (HEK 293T) were cultured in DMEM medium (Euroclone S.p.A., Pero, Milan, Italy, cat no ECM0102L) supplemented with $10 \%$ fetal bovine serum (FBS), $50 \mathrm{U} / \mathrm{mL}$ penicillin and $50 \mu \mathrm{g} / \mathrm{mL}$ streptomycin at $37^{\circ} \mathrm{C}$ in $5 \% \mathrm{CO}_{2}$. Cells were plated on eightchamber culture slides (Falcon cat no 354108) at a density of $2 \times 10^{4}$ cells $/ \mathrm{mL}$ per well. After $24 \mathrm{~h}$ of incubation, cells were treated with polymers alone or CNT/miRNA complexes. FAM-mir-503 (50 nM) was incubated with pure polyamines and polyamine-coated CNTs at different weight ratios (polymer/miRNA ratios of 2:1, 4:1, 8:1, 10:1 w/w and CNT/ miRNA 5:1, 10:1, 20:1 w/w, respectively) for $15 \mathrm{~min}$ at room temperature to allow complexation. Cells were incubated for $4 \mathrm{~h}$ with the complex formed by FAM-miR-503 and CNTs, washed with phosphate-buffered saline (PBS) and cultured for $24 \mathrm{~h}$. After $24 \mathrm{~h}$, cells were washed three times with cold PBS and fixed by incubation with a methanol/acetone (2:1) solution for $10 \mathrm{~min}$ at $-20^{\circ} \mathrm{C}$. After three PBS washes, cells were stained with $150 \mu \mathrm{L}$ of a solution of phalloidin-TRITC $(10 \mu \mathrm{g} / \mathrm{mL})$ for $1 \mathrm{~h}$, washed again and the nuclei stained with $150 \mu \mathrm{L}$ of intercalating dye Hoechst $33258(4 \mu \mathrm{g} / \mathrm{mL})$. Fixed cells were mounted with a glycerol/PBS solution (3:1) and kept covered to prevent dye photobleaching until fluorescence image acquisition.

\section{Confocal microscopy}

The confocal microscopy imaging was performed using an Olympus Fluoview FV1000 confocal microscope (Olympus Corporation, Tokyo, Japan), equipped with FV10-ASW version 4.2 software, Multi Ar (458-488 and $512 \mathrm{~nm}$ ), $2 \times \mathrm{He} / \mathrm{Ne}(543$ and $633 \mathrm{~nm}$ ) and $405 \mathrm{~nm}$ diode laser, using $60 \times$ (numerical aperture: 1.42 ) oil objective. Optical single sections were acquired with a scanning mode format of $1,024 \times 1,024$ pixels, sampling speed of $20 \mu \mathrm{s} /$ pixel and $12 \mathrm{bit} / \mathrm{pixel}$ images. Automated-sequential collection of multichannel images was performed in order to reduce spectral crosstalk between channels. Each group of images was processed and analyzed using the same settings (ie, laser power and detector amplification) and processed using Adobe Photoshop software (Adobe Systems Incorporated, San Jose, CA, USA).

\section{Preparation of polymer-coated BPs}

To test the properties of large CNT aggregates in 2D form (ie, sheets of BPs), we realized a home-made support by filtering a suspension of PAM-CNTs and comparing the obtained
BP with a commercial one (Buckeye Composites, Kettering, OH, USA) coated with PAMAM. Briefly, CNTs $(50 \mathrm{mg})$ were dispersed in distilled water $(15 \mathrm{~mL})$ containing PAMAM ( $\sim 30 \mathrm{mg}$ ). CNT suspensions were prepared as described previously. For BP preparation, the suspension of PAM-CNTs was filtered through a polyethersulfone filter $(0.22 \mu \mathrm{m}$, Sartorius Stedim Biotech, Göttingen, Germany). The membrane was dried at room temperature, placed on a film of agarose gel (3\%) in a Petri dish and sterilized under UV light. The PAM-BP was incubated with FAM-mir-503 (at a weight ratio of 10:1) for $15 \mathrm{~min}$. The commercial BP was cut into $5 \mathrm{~cm}$ strips and incubated with PAMAM as described earlier. Then, commercial PAMAM-coated BPs were cut into small pieces $\left(0.1 \mathrm{~cm}^{2}\right)$. Cells were seeded directly on the top of the membrane at a density of $6 \times 10^{4} / \mathrm{cm}^{2}$. After $24 \mathrm{~h}$, supports were treated with $4 \%$ paraformaldehyde at room temperature for $15 \mathrm{~min}$. Cells were stained for confocal microscopy as described earlier.

\section{Histological staining}

BPs were embedded in paraffin and cut into small slices $(5 \mu \mathrm{m})$. Sections were stained with hematoxylin and eosin (H\&E) and examined by optical microscopy (DM500; Leica Microsystems, Wetzlar, Germany).

\section{Statistical analysis}

Statistical comparison between various groups was performed by Student's $t$-test or one-way analysis of variance (ANOVA) with either least significant difference (LSD) post hoc tests, using the SPSS software (12.0.2). Comparisons were made between mean values from several experiments. Differences were considered significant when $p$-values were $<0.05$. Statistical significance is indicated with asterisk symbol for $p<0.05$.

\section{Results Sample preparation}

To study the different biophysical and biological properties (ie, nucleic acid binding, dimensions, toxicity and transfection ability) of polyamine-coated CNTs as a function of their aspect ratio (ie, dimensions) and evaluate their contribution in miRNA delivery to human cells, we prepared three different types of samples (ie, very small CNTs, medium-sized CNTs and large bidimensional sheets of CNTs, named "buckypapers"). To establish the optimal preparation condition, we modified the synthesis method reported in our previous paper $^{21}$ (Figure 1). Starting from a commercial batch of short $(0.5-2 \mu \mathrm{m})$ multiwalled CNTs, we suspended them into three different polymer solutions of PEI or PAMAM 


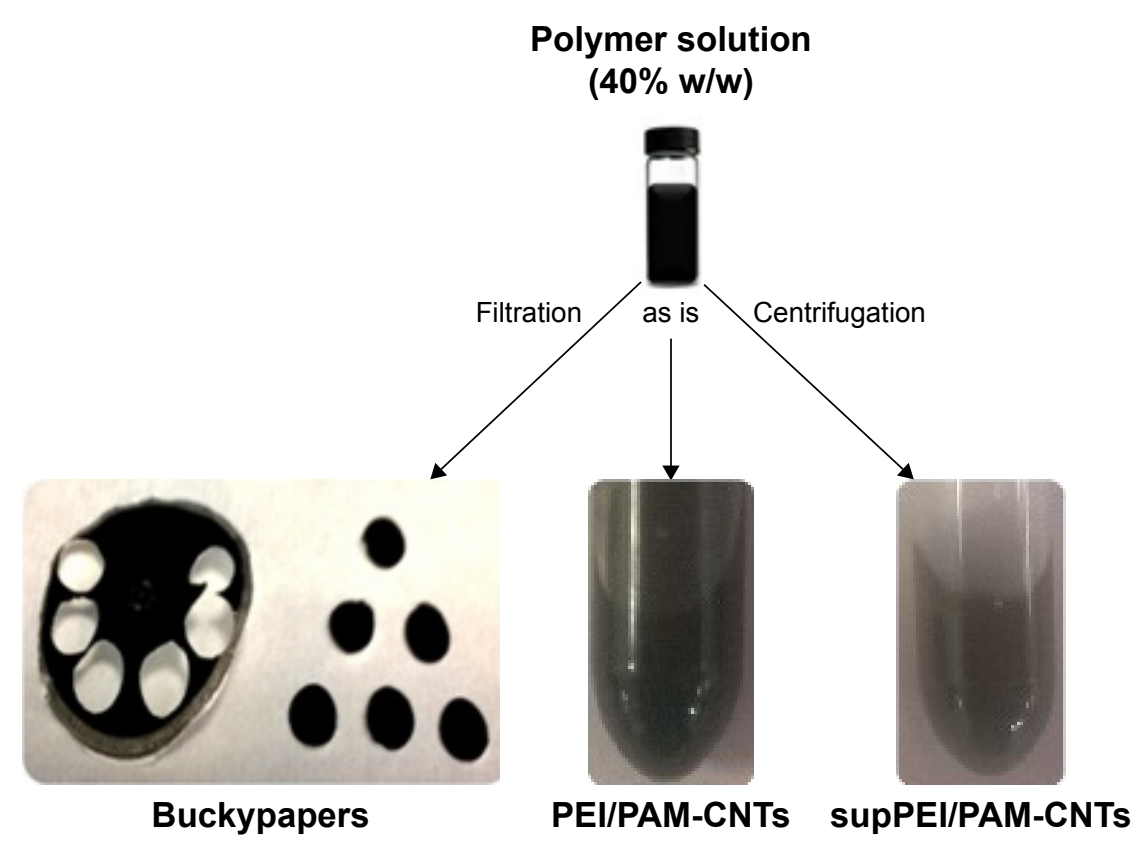

Figure I Schematic illustration of the general procedure for polymer-coated CNTs preparation (10\%, 20\% and 40\% w/w solution of PEI and PAMAM). Note: The most stable suspension (40\%) was used for the preparation of buckypapers and very small CNTs (supPEI/PAM-CNTs).

Abbreviations: CNTs, carbon nanotubes; PAMAM, polyamidoamine dendrimer; PEI, polyethyleneimine.

(at a concentration of $10 \%, 20 \%$ and $40 \%, \mathrm{w} / \mathrm{w}$, respectively). Only the $40 \% \mathrm{w} / \mathrm{w}$ solution afforded products (PEI-CNTs and PAM-CNTs) that resulted to be stable for many weeks. The other solutions $(10 \%$ and $20 \%, \mathrm{w} / \mathrm{w})$ prevented the formation of stable suspensions of CNTs. In fact, after few minutes from initial incubation a great amount of CNT aggregates was visible at the bottom of each vial (Figure S1) and were not considered for further experiments. We hypothesize that this behavior can be explained by considering that the amount of polymer in the $10 \%$ and $20 \%$ solutions is not enough to completely bind CNTs and resuspend them.

In any case, we observed that after few weeks from the preparation of stable CNT suspensions, a precipitation of large nanotube aggregates took place. Interestingly, in our previous paper we found that after prolonged centrifugation of CNT suspensions, a certain amount of nanotubes, likely the smallest ones, remained in the supernatant. Therefore, to characterize the properties of these two fractions (ie, aggregate and small CNTs) and assess their effectiveness to deliver miRNAs and contribute to the overall transfection efficiency, we decided to filter a part of the initial suspension to isolate the larger aggregates and retain the very small CNTs (coated with polymers) contained in the supernatant (Figure 1). The filtration of CNTs afforded a bidimensional product, commonly referred to as "buckypaper" for its resemblance to a sheet of paper. A third part consisting of a stable suspension of polymer-coated CNTs was used "as is" (Figure 1).

\section{Quantification and localization of polyamine polymers on CNTs}

The amount of polyamine polymers adsorbed on CNTs was measured by TGA. The percentage (in weight) of the polymers adsorbed on CNTs was $9.83 \% \pm 2.18 \%$ for PEI and $21.36 \% \pm 3.57 \%$ for PAMAM. We reasoned that in order for the CNTs to absorb polymers, the hydrophobic part of the polyamine polymer should interact with the hydrophobic part of CNTs, and the overall effect is the deposition of a thin layer of polymer on CNTs, as shown by TEM (Figure 2).

\section{Biophysical characterization of supernatants containing very small polymer-coated CNTs}

The suspensions of polymer-coated CNTs are generally a mixture of large, medium and small CNTs. Their aspect ratio prevents the dimensional characterization by traditional DLS technique because CNTs are not spherical particles. Therefore, DLS can be employed only to measure very small nanotubes, assuming their "sphericity". For these reasons, we characterized only the polymer-coated CNT fraction obtained after centrifugation (the supernatant), which contains the smallest compounds. In particular, we characterized two supernatant samples, supPEI-CNTs and supPAM-CNTs, and we analyzed their size and $\zeta$-potential at different time points $(0,12 \mathrm{~h}$ and 20 days). 


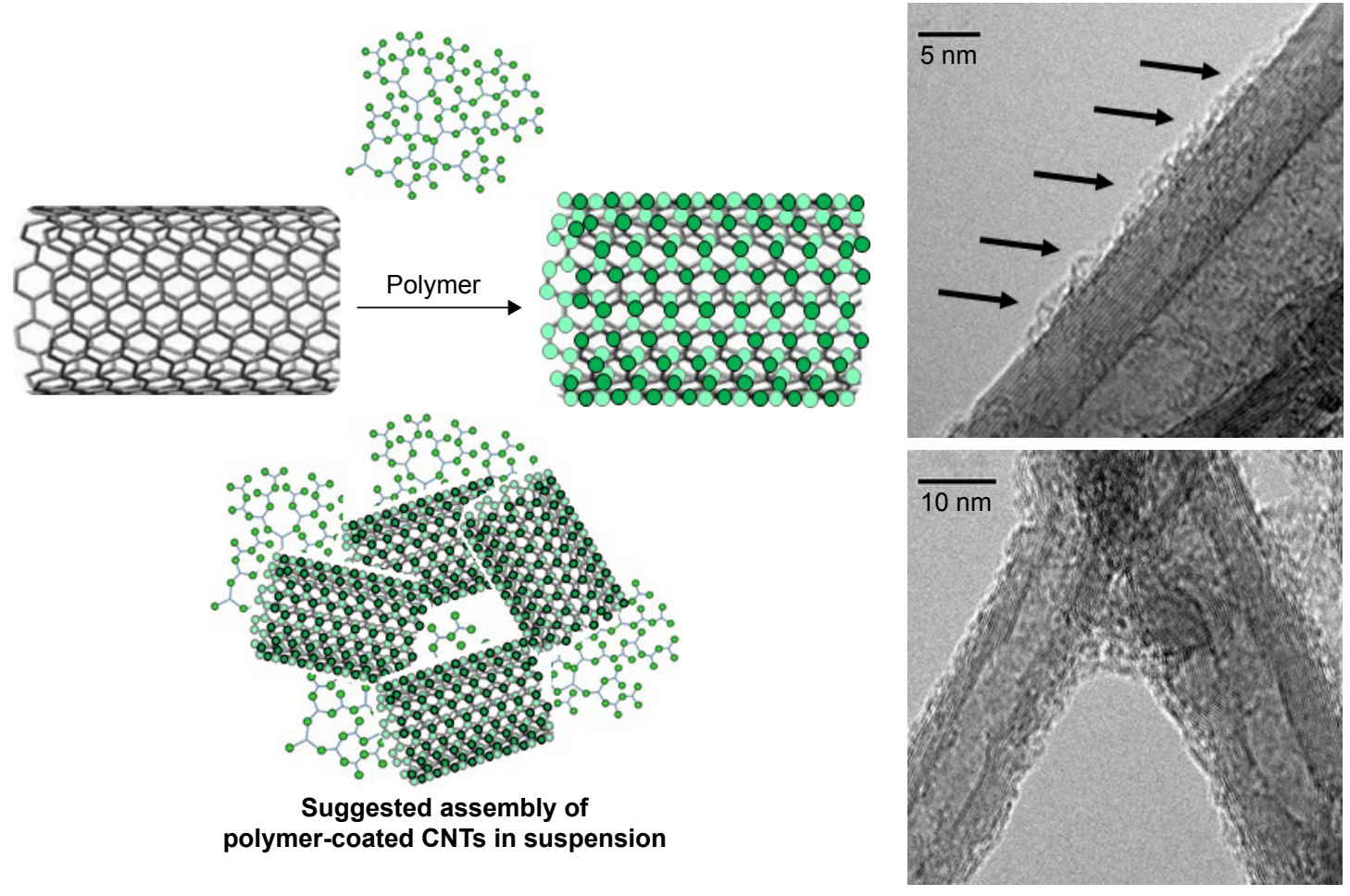

Figure 2 Schematic representation of the hypothetical binding of polyamine polymer to the surface of CNTs.

Notes: The thin layer of PAMAM polymer is visible by transmission electron microscopy as a cloudy matter absorbed on the CNT's surface (black arrows). Aggregates of CNTs indicate that the polyamine polymer is able to interact with multiple CNTs leading to a complex and not homogeneous mixture.

Abbreviations: CNTs, carbon nanotubes; PAMAM, polyamidoamine dendrimer.

The dimensions of supPEI-CNTs and supPAM-CNTs are quite similar at $t=0$ (Table 1 ). In supPEI-CNTs, the presence of a population of small compounds sized $19.9 \pm 0.9 \mathrm{~nm}$ increased the polydispersity index (PDI) compared to supPAM-CNTs. We also measured the $\zeta$-potential of the two compounds as a function of time (Table 2). $\zeta$-Potential is correlated with the colloidal stability of the system under investigation; the higher its value the higher is the

Table I Hydrodynamic mean diameter and polydispersity index (PDI) of polymer-coated CNT supernatants evaluated by two different and complementary methods, NNLS analysis of size distribution and Cumulant analysis

\begin{tabular}{|c|c|c|c|c|}
\hline \multirow[t]{2}{*}{ Samples } & \multicolumn{3}{|c|}{$\begin{array}{l}\text { Hydrodynamic diameter } \\
(\mathrm{nm})\end{array}$} & \multirow[t]{2}{*}{ PDI } \\
\hline & $\begin{array}{l}\text { NNLS pe } \\
\text { mean }\end{array}$ & & $\begin{array}{l}\text { Cumulant } \\
\text { Z-average }\end{array}$ & \\
\hline supPEI-CNTs $(t=0)$ & $19.9 \pm 0.9$ & $213 \pm 19$ & $148.1 \pm 0.9$ & $0.477 \pm 0.005$ \\
\hline supPAM-CNTs $(t=0)$ & 0 & $195 \pm 18$ & $187.0 \pm 1.0$ & $0.272 \pm 0.003$ \\
\hline supPEI-CNTs ( $t=20 \mathrm{~d})$ & $25.7 \pm 6.2$ & $340 \pm 86$ & $203.5 \pm 0.9$ & $0.553 \pm 0.004$ \\
\hline supPAM-CNTs $(t=20 \mathrm{~d})$ & 0 & $192 \pm 17$ & $183.1 \pm 0.9$ & $0.269 \pm 0.009$ \\
\hline
\end{tabular}

Note: Values were reported as mean value \pm standard deviation on three repeated measurements.

Abbreviations: BP, buckypaper; CNTs, carbon nanotubes; PDI, polydispersity index; PEl, polyethyleneimine. stability of the compounds. We found that the $\zeta$-potential of supPEI-CNTs increased from $9.0 \pm 0.8$ to $10.5 \pm 1.7 \mathrm{mV}$ (in 20 days) but decreased in supPAM-CNTs (from $31.5 \pm 0.7$ to $20.2 \pm 1.2 \mathrm{mV}$ ). The $\zeta$-potential of supPEICNTs was always lower than that of supPAM-CNTs. This is in agreement with the slow precipitation behavior of the two compounds that we observed. Moreover, in the same timeframe, the size of supPEI-CNTs increased about $50 \%$ (from $148.1 \pm 0.9$ to $203.5 \pm 0.9 \mathrm{~nm}$ ), whereas the size of

Table 2 To study aggregation phenomena, electrophoretic mobility $(\mu \mathrm{mcm} / \mathrm{Vs})$ has been measured to get $\zeta$-potential $(\mathrm{mV})$ of polymer-coated CNT supernatants at $t=0$, after 12 and $20 \mathrm{~h}$ from the initial preparation

\begin{tabular}{lllll}
\hline Samples & Time, $t$ & $\begin{array}{l}\text { Mobility } \\
(\mu \mathrm{m} \mathrm{cm} / \mathbf{V s})\end{array}$ & $\begin{array}{l}\zeta \text {-potential } \\
(\mathbf{m V})\end{array}$ & $\begin{array}{l}\text { Conductivity } \\
(\mathrm{mS} / \mathrm{cm})\end{array}$ \\
\hline supPEI-CNTs & 0 & $0.7 \mathrm{I} \pm 0.06$ & $9.0 \pm 0.8$ & $0.143 \pm 0.005$ \\
& $12 \mathrm{~h}$ & $0.81 \pm 0.01$ & $10.4 \pm 0.1$ & $0.145 \pm 0.001$ \\
& 20 days & $1.21 \pm 0.13$ & $10.5 \pm 1.7$ & $0.191 \pm 0.002$ \\
supPAM-CNTs & 0 & $2.47 \pm 0.05$ & $31.5 \pm 0.7$ & $0.108 \pm 0.005$ \\
& $12 \mathrm{~h}$ & $1.88 \pm 0.14$ & $24.0 \pm 1.8$ & $0.117 \pm 0.005$ \\
& $20 \mathrm{~d}$ & $1.59 \pm 0.09$ & $20.2 \pm 1.2$ & $0.140 \pm 0.005$ \\
\hline
\end{tabular}

Note: Preliminary to the mobility measurement, also conductivity $(\mathrm{mS} / \mathrm{cm})$ of the sample was determined within the same cuvette.

Abbreviations: CNTs, carbon nanotubes; PEI, polyethyleneimine. 
supPAM-CNTs remained almost constant (from 187.0 \pm 1.0 to $183.1 \pm 0.9 \mathrm{~nm}$ ) (Table 2).

Interestingly, after 10 days the conductivity of the two samples increased, which indicated an increasing amount of charged species in the solution, likely due to polymer degradation or a possible detachment of the polyamine polymer from CNTs. However, this phenomenon has not been investigated completely in this work and deserves further studies.

\section{Gel retardation assays}

To assess the ability of polyamine-coated CNTs to bind small nucleic acids (ie, oligonucleotides, miRNA mimic, etc), we performed agarose gel retardation assays (Figure 3 ). Both polyamine-coated CNTs were able to bind efficiently a 70 nt oligo mimicking a pre-miRNA (hsa-mir-503) even at the lowest CNTs/miRNA weight ratio (ie, 2:1) (Figure 3A). Both PEI and PAMAM polymers formed large aggregates with the miRNA that were unable to migrate into the gel, in agreement with data already reported. ${ }^{21,43}$ Similarly, the complete formation of PEI-CNTs and PAMAM-CNTs complexes occurred at a higher weight ratio of 10:1 (lane 8) and 20:1 (lane 16), respectively. The higher amount of PAMCNTs needed to bind completely the oligo suggests that this compound could bind the nucleic acid less tightly. Moreover, by comparing the fluorescence signal of PEI and PAMAM complexes within the wells of the agarose gel (lanes 3-6 and 10-13, respectively), a more intense signal was observed for PAMAM compared to PEI. Interestingly, this might
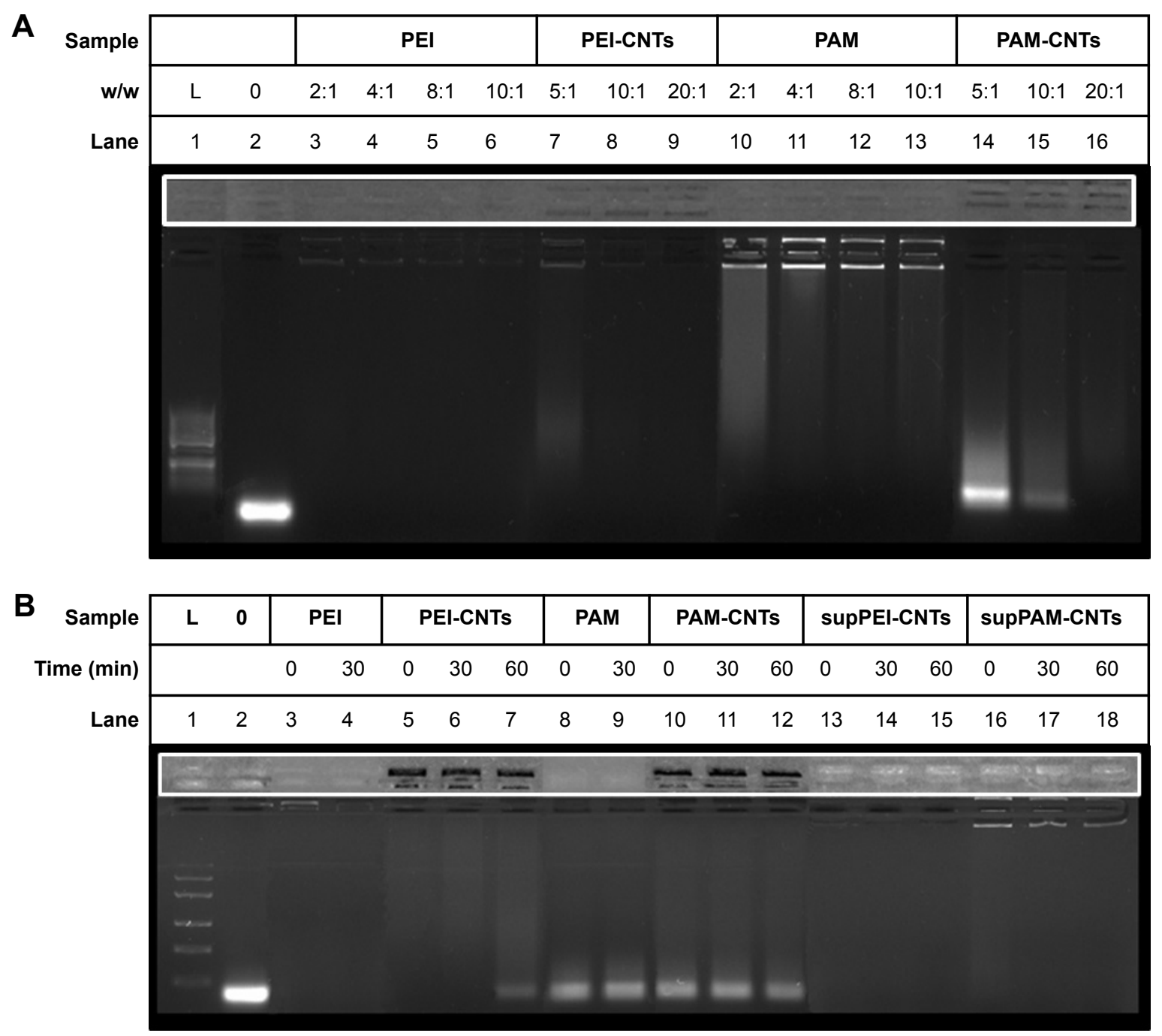

Figure 3 Agarose gel electrophoresis of polymer-coated CNTs complexed with a synthetic DNA.

Notes: Agarose gel (1\%) stained with Hoechst 33258 shows that (A) PEl-coated and PAMAM-coated CNTs are able to bind DNA (I0:I w/w) (lanes 7-9 and I4-16) similar to starting polymers (lanes 3-6 and 10-13) but at a different weight ratio. DNA ladder (50 bp) was loaded in lane I and a well-characterized DNA fragment ( 250 bP) in lane 2. The heparin competitive assay (B) indicates that the nucleic acid bound to PAM-CNTs is more easily released (lanes I0-I2), compared to PEI-CNTs (lanes 5-7 and 13-15), whereas supPEI-CNTs and supPAM-CNTs can bind nucleic acid more strongly compared to their CNT counterparts.

Abbreviations: CNTs, carbon nanotubes; CTR, control; PAMAM, polyamidoamine dendrimer; PEI, polyethyleneimine. 
indicate a different kind of interaction and complexation (ie, minor- or major-groove binding) between the polymer and the oligonucleotide. We have observed previously that the minor groove binding by polyamine polymers prevents, at least in part if not completely, the fluorescence quenching of the intercalating dye. ${ }^{43}$ The tighter interaction of PEI and PEICNTs with DNA compared to PAMAM was also confirmed by the heparin competition assay. ${ }^{44}$ This assay consists in the incubation of polymer-coated CNTs complexed with oligonucleotides with a negatively charged high-molecular weight macromolecule (ie, heparin), which could electrostatically compete with the oligonucleotide and displace it (Figure 3B). PEI (lanes 3 and 4) and PEI-CNTs (lanes 5-7) bind the oligonucleotide stronger than PAMAM (lanes 8 and 9) and/ or PAMAM-CNTs (lanes 10-12), as indicated by the presence of the free oligo band. On the contrary, supPEI-CNTs and supPAM-CNTs form strong and very stable complexes (at the same weight ratio of PEI-CNTs or PAM-CNTs) with the oligonucleotide. In fact, heparin was not able to displace the complexed oligo even after $60 \mathrm{~min}$ incubation (Figure 3B, lanes 13-15 and 16-18). This result suggests that the length of CNTs might have an effect on both nucleic acid binding process and stabilization of the complex.

\section{Cytotoxicity of polyamine-coated CNTs Cytotoxicity of PEI-CNTs and PAM-CNTs}

To evaluate the cytotoxicity of polyamine-coated CNTs, HEK 293T cells were incubated with increasing amount of polymers (PEI or PAMAM) in the range of $0.5-40 \mu \mathrm{g} / \mathrm{mL}$ and polymer-CNTs in the range of $10-50 \mu \mathrm{g} / \mathrm{mL}$ (Figure 4 ). For pure polymers, the cell viability decreased at higher concentrations (Figure 4A). PEI was relatively toxic at a
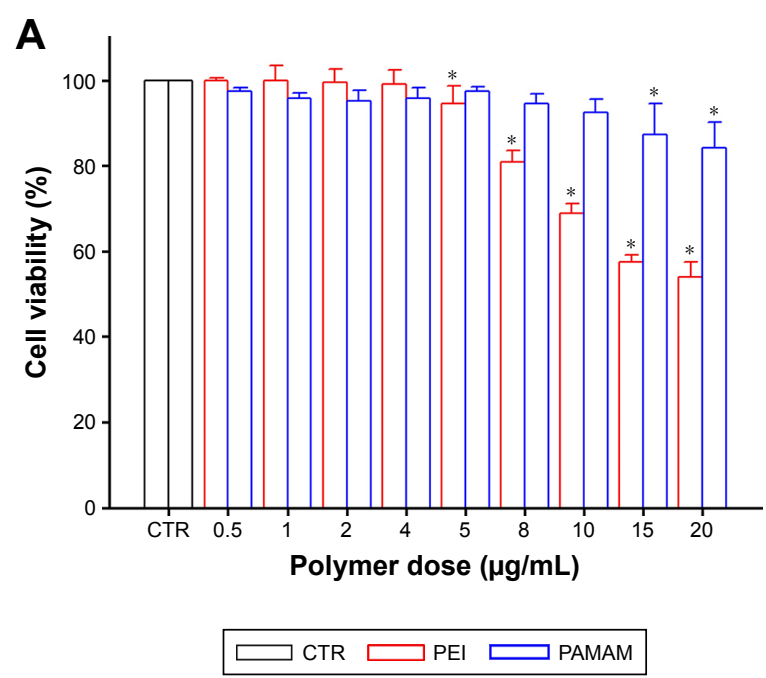
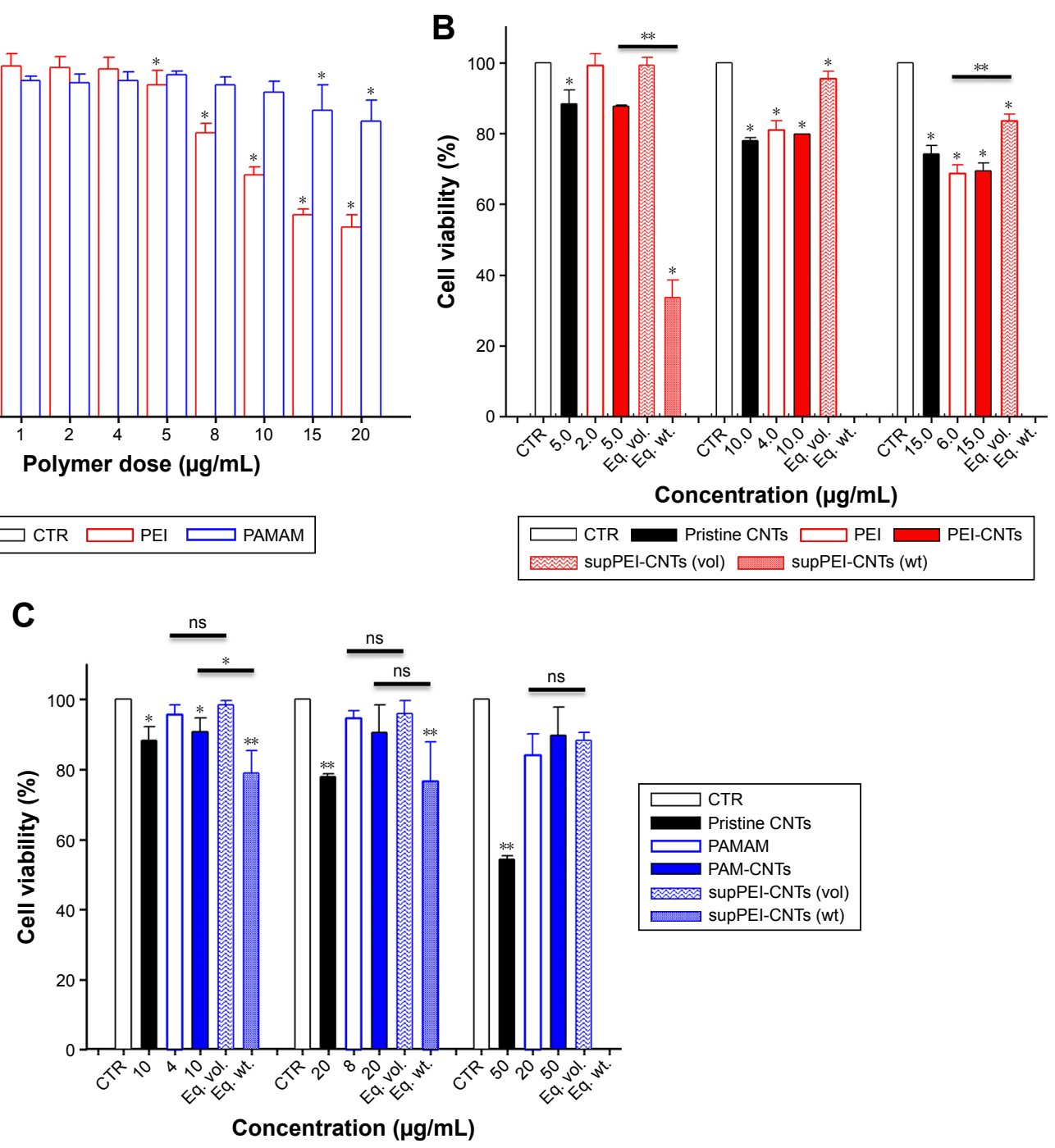

Figure 4 Cytotoxicity assay after 24-h treatment with (A) PEI (red) and PAMAM (blue) polymers and CNTs functionalized with (B) PEI or (C) PAMAM. Notes: Values are expressed as mean $\pm \mathrm{SE}(\mathrm{n}=5)(* \mathrm{P}<0.05$, $* * \mathrm{P}<0.0 \mathrm{I})$.

Abbreviations: CNTs, carbon nanotubes; ns, not significant; PAMAM, polyamidoamine dendrimer; PEI, polyethyleneimine; vol, volume; wt, weight. 
concentration of $10 \mu \mathrm{g} / \mathrm{mL}$ (cell viability $=69.8 \% \pm 2.3 \%$ ), whereas induced a significant cell death $(53.9 \% \pm 3.5 \%)$ at $20 \mu \mathrm{g} / \mathrm{mL}$. On the contrary, PAMAM was less toxic compared to PEI. At $10 \mu \mathrm{g} / \mathrm{mL}$, the cell viability was $92.4 \% \pm 3.2 \%$, whereas even at $20 \mu \mathrm{g} / \mathrm{mL}$ the cell viability remained quite high $(84.2 \% \pm 6.0 \%)$. Only at a higher concentration $(>40 \mu \mathrm{g} / \mathrm{mL})$ PAMAM induced a significant cell death $(66.1 \% \pm 6.6 \%)$ (data not shown).

Similarly, we treated HEK 293 T cells with PEI-CNTs $(10-30 \mu \mathrm{g} / \mathrm{mL})$ and PAMAM-CNTs $(10-50 \mu \mathrm{g} / \mathrm{mL})$. For PEICNTs, the overall toxicity parallels that of pure polyamine or uncoated CNTs (especially at $10 \mu \mathrm{g} / \mathrm{mL}$ ), whereas for PAMAM-CNTs the overall toxicity seems dependent only on the polymer amount (ie, at $50 \mu \mathrm{g} / \mathrm{mL}$ the toxicity of PAMAM-CNTs is $89.8 \% \pm 8.0 \%$, similar to $84.2 \% \pm 6.0 \%$ of PAMAM, whereas that of CNTs is $54.3 \% \pm 1.0 \%$ ). This indicates that the coating with PAMAM is able to decrease significantly the toxicity of the pristine CNTs.

\section{Cytotoxicity of supCNTs}

To evaluate the cytotoxicity of very small CNT preparations, we treated HEK 293 T cells with different amounts of supPEICNTs and supPAM-CNTs. In particular, to compare the toxicity of supernatants with that of pure polymers we incubated cells with $4,8,12$ or $20 \mu \mathrm{L}$ of the suspension, which corresponded to the same amount (in volume) of pure polymers. Moreover, in order to compare the toxicity of supernatants with that of polyamine-coated CNTs, we treated cells by using the same amount (in weight) of CNTs $(10,20,30$ or $50 \mu \mathrm{g})$ (Figure 4). The amount of CNTs contained in supernatants was determined by spectrophotometry (Figure S2).

By using the same amount in volume of supPEI-CNTs, we found that these compounds were not particularly toxic even at the highest concentration tested (viability $=83.5 \% \pm 2.0 \%$ ) (Figure 4B) compared to pure PEI polymer. At this concentration, supPEI-CNTs were less toxic than pure PEI polymer. On the contrary, by using the same amount in weight of supPEI-CNTs, we found that these compounds were extremely toxic even at the lowest amount $(10 \mu \mathrm{g})$ $(33.7 \% \pm 4.8 \%)$ compared to PEI-CNTs. We have no cytotoxicity data for higher amounts (20 and $30 \mu \mathrm{g})$, since we realized that the volume required to perform the assay was too big (not available or not compatible with culture conditions). It is likely that the high toxicity displayed by supPEI-CNTs is due to the dimension of nanotubes, as the amount of free polymer or residual CNTs in suspension is relatively low and do not justify such reduced viability.

Similar to PAM-CNTs, supPAM-CNTs also are relatively not toxic (Figure 4C) in all of the conditions analyzed.
Compared to the pure polymer, supPAM-CNTs even at the highest amount $(20 \mu \mathrm{L})$ were not toxic $(88.3 \% \pm 2.2 \%)$. Interestingly, $20 \mu \mathrm{g}$ of supPAM-CNTs induces a toxicity that is only a little bit higher compared to that obtained treating HEK 293 T cells with the same amount of PAM-CNTs (Figure 4C). Once again, the toxicity data of supernatant CNTs confirm that the use of PAMAM to coat CNTs is preferable compared to PEI.

\section{Cytotoxicity of BPs}

To assess the toxicity of BPs, small pieces of BP coated with PAMAM (PAM-BPs) were used to grow cells. Interestingly, the amount of PAMAM adsorbed by BPs is lower $(8.87 \% \pm 1.55 \% \mathrm{w} / \mathrm{w})$ than that absorbed by PAM-CNTs. This can be explained by considering the different morphology of the $2 \mathrm{D}$ material and the different surface area (or aspect ratio) of the two materials. In fact, BPs are more compact compared to CNTs, and therefore the BP surface is less "exposed" to the solution where, on the contrary, CNTs are more free to float and bind the polymer (higher degree of freedom in solution). After cell growth, we noticed that many cells remained stacked to the surface even after trypsin treatment, and it was not possible to detach them and perform the assay determination. Moreover, MTT assay was not suited to quantify viable cells in BPs owing to the oxidation of the substrate (MTT) by reactive CNTs during the measurement. ${ }^{42}$ This induces a rapid coloration of the solution which prevented a correct cytotoxicity assessment by this assay.

\section{Cellular uptake of a fluorescent microRNA mimic (FAM-mir-503) Delivery mediated by PEI-CNTs and PAM-CNTs}

In order to determine the effectiveness of our compounds to deliver miRNA mimics into mammalian cells, we treated HEK 293 T cells with different amounts of polyamine-coated CNTs complexed with a fluorescent microRNA mimic (FAM-mir-503) (CNT/oligo ratios of 5:1, 10:1, 20:1 and $25: 1 \mathrm{w} / \mathrm{w}$ ) and polyamine complexes (polymer/oligo ratios of $2: 1,4: 1,8: 1$ and 10:1,w/w). The delivery was evaluated $24 \mathrm{~h}$ post-transfection by confocal fluorescence microscopy. PEI and PAMAM displayed an optimal transfection efficiency occurring at CNT/oligo ratios of 8:1 and 10:1 w/w, respectively (Figures S3 and S4). PAMAM displayed a higher transfection efficiency compared to PEI. Similarly, PEI-CNTs and PAM-CNTs displayed a good transfection efficiency at CNT/oligo ratios of 10:1 and 20:1 w/w, respectively (Figures S5 and S6). Similar to pure polymer, PAMCNTs were more efficient than PEI-CNTs to transfect FAMmir-503 (Figure 5). 
Blank

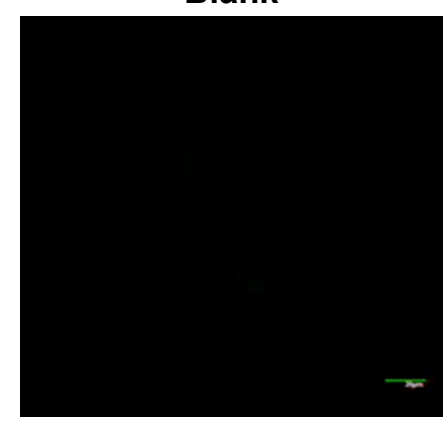

Hoechst

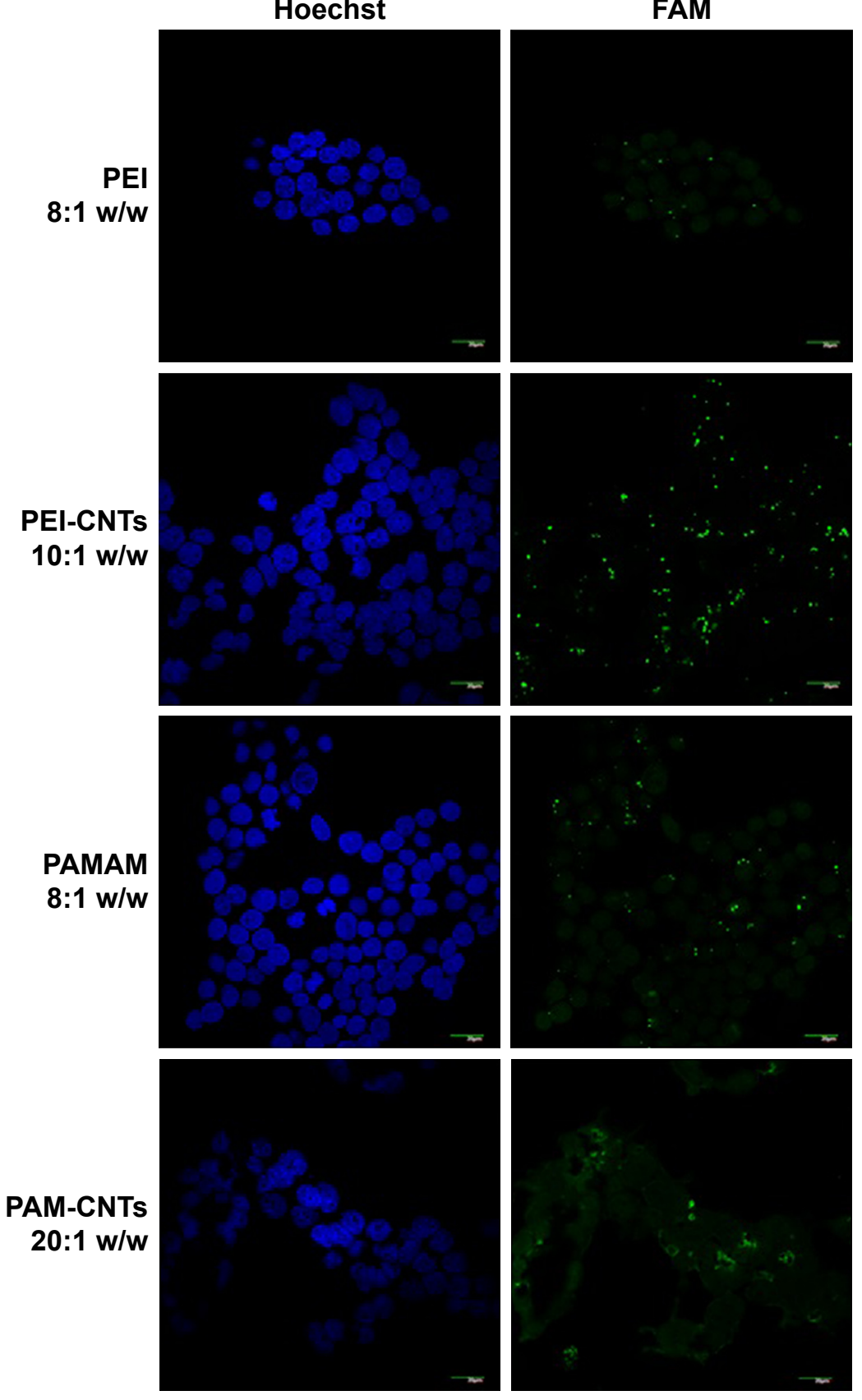

Figure 5 Fluorescence images of Hela cells after 24-h treatment with pure polymers and polymer-coated CNTs complexed with FAM-mir-503, at different weight ratios. Notes: A brighter fluorescence of PAM-CNTs (35.8\%) compared to PEI-CNTs (16.9\%) indicates an increased transfection ability of the dendrimer-coated CNTs (Figure S7 for quantitative data). The pure polymers PEI and PAMAM displayed a fluorescence intensity of $6.1 \%$ and $26.9 \%$, respectively (compared to the blank control). Magnification $60 \times$, scale bars represent $20 \mu \mathrm{m}$.

Abbreviations: CNTs, carbon nanotubes; PAMAM, polyamidoamine dendrimer; PEI, polyethyleneimine.

\section{Delivery mediated by supernatants of} polyamine-coated CNTs

To evaluate the uptake efficiency of mir-503 mimic by supernatants of polyamine-coated CNTs, we treated HEK 293T cells with different amounts of supernatants from polyamine-coated CNTs complexed with FAM-mir-503. In particular, to compare the efficiency of supernatants to that of pure polymers, complexes were formed by incubating $0.4 \mu \mathrm{g}$ of FAM-mir- 503 with
$8 \mu \mathrm{L}$ of supPEI-CNTs or supPAM-CNTs, which correspond to the same amount (in microliters) of pure polymers used before. To compare the efficiency of supernatants to that of polyaminecoated CNTs, complexes were formed by incubating $0.4 \mu \mathrm{g}$ of FAM-mir-503 with an amount of $120 \mu \mathrm{L}$ for supPEI-CNTs or supPAM-CNTs, which correspond to the same amount (in micrograms) of polyamine-coated CNTs used before. We observed (Figure 6) that the intracellular fluorescence is 


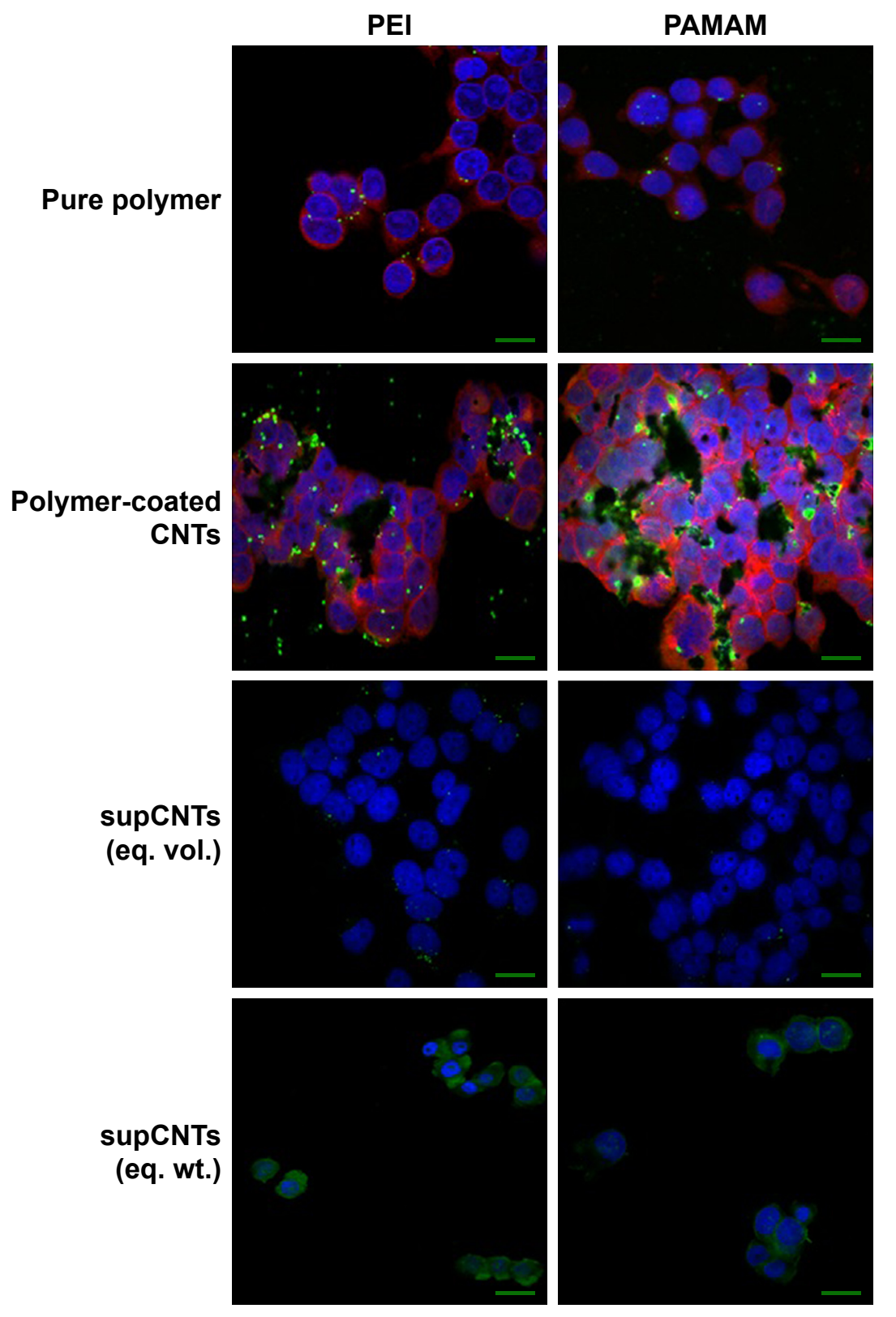

Figure 6 Fluorescence images of Hela cells after 24-h treatment transfected with pure polymers (PEI and PAMAM), polymer-coated CNTs and supernatants (supPEI-CNTs [vol] and supPEI-CNTs [wt]) complexed with FAM-mir-503 at 10:I weight ratio.

Note: Magnification 60x, scale bars represent $20 \mu \mathrm{m}$.

Abbreviations: CNTs, carbon nanotubes; PAMAM, polyamidoamine dendrimer; PEl, polyethyleneimine; vol, volume; wt, weight.

weak when cells were treated with supernatants at the same pure polymers concentration, whereas a significantly increased uptake was visible at the same polyamine-coated CNT concentration, although we also observed an increased cytotoxicity. However, we did not observe a significant delivery of the bound miRNA, likely due to the high stability of the complex, as observed in agarose gel experiments. Overall, these data indicate that the amounts of polyamine-coated CNTs or the free polymers are negligible and are not enough to contribute to an efficient delivery of miRNA mimics.

\section{Delivery mediated by bidimensional BP}

To evaluate the uptake efficiency of mir-503 mimic by bidimensional sheets of CNTs (ie, BPs), we prepared a flexible support made by PAMAM-coated CNTs on which cells were let to grow. For comparison purposes, we performed the same experiment with a commercial BP. This experiment allowed us to study the ability of huge aggregates of CNTs to transfect cells as "nanoneedles" through a mechanism generally referred to as "piercing", ${ }^{45}$ which consists in the passive penetration of the cell membrane by long CNTs (or parts 

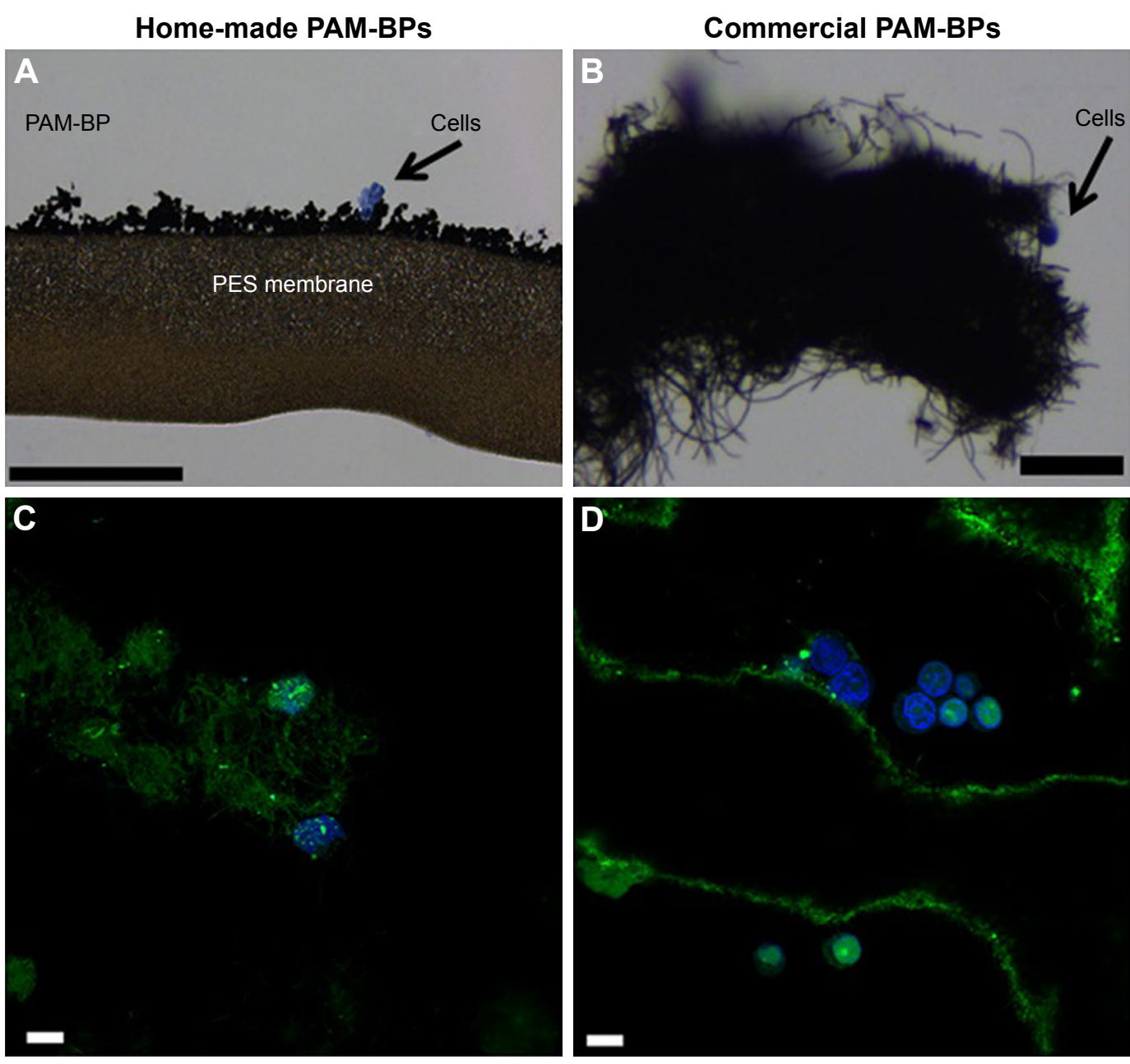

Figure 7 Cells grown on buckypapers.

Notes: Hematoxylin/eosin staining of cells grown on $(\mathbf{A})$ home-made BP (scale bar $=200 \mu \mathrm{m})$ and $(\mathbf{B})$ commercial BP $($ scale bar $=25 \mu \mathrm{m})$. The commercial PAM-BP incubated with FAM-mir-503 and visualized by confocal microscopy after cell culture (C and D). The green bundles show the intricate network of CNTs forming the BP, whereas the green lines represent the irregular layers of BP. The nuclear staining indicates the presence of healthy cells, whereas the green spots within them indicate that these rigid bidimensional substrates are able to penetrate cells and deliver their nucleic acid cargo. (Scale bar $=20 \mu \mathrm{m}$.)

Abbreviations: BP, buckypaper; CNTs, carbon nanotubes.

of them) and not through classic endocytic mechanisms (ie, clathrin-mediated or caveolae-mediated pathways).

We observed the BP layer after having cultured cells on it (Figure 7A), and found only a few cells adhering on the surface. The commercial BP made by longer CNTs was thicker than our home-made BP (Figure 7B). Anyway, we were able to visualize few cells grown on it by H\&E staining. This suggested that PAMAM-coated BP might be a suitable substrate for culturing cells without appreciable cytotoxicity. To investigate the binding efficiency of this bidimensional substrate, the commercial BP was coated with PAMAM and incubated with FAM-mir-503. Confocal microscopy (Figure 7C-E) revealed the intricate network of CNTs formed with the BP (the green bundles in Figure 7C) and the irregular layers that the BP is made of (the green lines in Figure 7D and E). Nuclear staining is suggestive of healthy cells, whereas the green spots within them indicate that these rigid bidimensional substrates are able to enter into cells and likely deliver miRNAs. However, further studies are needed to investigate better the binding of miRNA mimic and the kinetic of delivery that seem different compared to that observed with CNT suspensions.

\section{Discussion}

The characterization of the biophysical and biological properties of polyamine-coated CNTs and bidimensional BPs and the precise evaluation of their contributions in microRNA delivery to human cells are important aspects for the optimization of delivery vectors. Our study was aimed at studying various structural and biophysical aspects of polyamine-coated CNTs that are generally underevaluated in many studies. However, several studies emphasized that 
these contributions may affect the overall efficiency of delivery vectors. ${ }^{43,46,47}$

In our work, we studied how the size of two polyaminecoated CNTs may influence toxicity and transfection efficiency and ultimately impair the delivery of microRNAs to human cells. We found that the nominal size of commercial small CNTs (ie, $0.5-2 \mu \mathrm{m}$ in our case) is actually not representative of all the possible compounds present in the sample. In fact, we found a fraction of very short CNTs within the compound suspensions that have different properties compared to longer CNTs. We also found that longer CNTs may transfect cells, despite their dimension, by exploiting their "nanoneedle" aspect and "piercing" cells effectively.

Apart from the dimension of CNTs, we also found that CNTs coated with different polyamines display essentially the characteristics of the polymer used to coat them. Therefore, it is possible to obtain efficient polymer-coated CNT-based delivery vectors by simply employing polymers with low toxicity and high transfection properties, like PEI and PAMAM. These polymers form a tiny layer over the hydrophobic surface of CNTs that are in any case able to bind oligonucleotides such as the miRNA mimic we have designed.

Interestingly, we also found that bidimensional sheets of CNTs (ie, BPs) can be efficiently prepared starting from CNT suspensions by a straightforward filtration step. We found that these bidimensional BPs are versatile supports for cell cultures once functionalized to reduce their hydrophobicity. The coating of BPs with PAMAM allowed us to obtain hydrophilic sheets of nested CNTs that can be used to culture cells for longer periods without any drawbacks.

Finally, we demonstrated that each of the three main components of a standard suspension of polymer-coated CNTs (ie, very short, medium-sized and very long CNTs) are able to contribute differently to miRNA transfection. Therefore, we think that our investigation may be useful to help researchers involved in modulating gene expression to choose or optimize the transfection efficiency of miRNA delivery by CNT-based vectors.

\section{Conclusion}

The characterization of the biophysical and biological properties of polyamine-coated CNTs and bidimensional BPs allowed us to precisely evaluate their contributions in microRNA delivery to human cells. This is an important aspect for the optimization of delivery vectors. Here, we studied how two polyamine-coated CNT systems may influence the toxicity and the transfection efficiency of
microRNAs to human cells. We found that a fraction of very short CNTs within the compound suspensions has different properties compared to longer CNTs, and longer CNTs may transfect cells by "piercing" cells effectively, ultimately contributing to overall transfection. Interestingly, we also found that bidimensional sheets of CNTs (ie, BPs) are versatile supports for cell cultures, and when properly functionalized they have a reduced toxicity. In conclusion, we think that our investigation will be useful to researchers interested to modulate the gene expression of human cells through the delivery of microRNAs by CNT-based vectors.

\section{Disclosure}

The authors report no conflicts of interest in this work.

\section{References}

1. Andrews R, Weisenberger MC. Carbon nanotube polymer composites. Curr Opin Solid State Mater Sci. 2004;8:31.

2. Harrison BS, Atala A. Carbon nanotube applications for tissue engineering. Biomaterials. 2007;28(2):344-353.

3. Masotti A, Caporali A. Preparation of magnetic carbon nanotubes (Mag-CNTs) for biomedical and biotechnological applications. Int J Mol Sci. 2013;14(12):24619-24642.

4. Park S, Kang YJ, Majd S. A review of patterned organic bioelectronic materials and their biomedical applications. Adv Mater. 2015;27(46): 7583-7619.

5. Lacerda L, Raffa V, Prato M, Bianco A, Kostarelos K. Cell-penetrating carbon nanotubes in the delivery of therapeutics. NanoToday. 2007; 2(6):38-43.

6. Park S, Kim YS, Kim WB, Jon S. Carbon nanosyringe array as a platform for intracellular delivery. Nano Lett. 2009;9(4):1325-1329.

7. Nunes A, Amsharov N, Guo C, et al. Hybrid polymer-grafted multiwalled carbon nanotubes for in vitro gene delivery. Small. 2010;6(20): 2281-2291.

8. Siu KS, Zheng X, Liu Y, et al. Single-walled carbon nanotubes noncovalently functionalized with lipid modified polyethylenimine for siRNA delivery in vitro and in vivo. Bioconjug Chem. 2014;25(10): 1744-1751.

9. Iannazzo D, Piperno A, Pistone A, Grassi G, Galvagno S. Recent advances in carbon nanotubes as delivery systems for anticancer drugs. Curr Med Chem. 2013;20(11):1333-1354.

10. Zhang W, Zhang Z, Zhang Y. The application of carbon nanotubes in target drug delivery systems for cancer therapies. Nanoscale Res Lett. 2011;6:555.

11. Pantarotto D, Singh R, McCarthy D, et al. Functionalized carbon nanotubes for plasmid DNA gene delivery. Angew Chem Int Ed Engl. 2004;43(39):5242-5246.

12. Singh R, Pantarotto D, McCarthy D, et al. Binding and condensation of plasmid DNA onto functionalized carbon nanotubes: toward the construction of nanotube-based gene delivery vectors. J Am Chem Soc. 2005;127(12):4388-4396.

13. Liu Y, Wu DC, Zhang WD, et al. Polyethylenimine-grafted multiwalled carbon nanotubes for secure noncovalent immobilization and efficient delivery of DNA. Angew Chem Int Ed Engl. 2005;44(30): 4782-4785.

14. Liu M, Chen B, Xue Y, et al. Polyamidoamine-grafted multiwalled carbon nanotubes for gene delivery: synthesis, transfection and intracellular trafficking. Bioconjug Chem. 2011;22(11):2237-2243.

15. Nagaraju K, Reddy R, Reddy N. A review on protein functionalized carbon nanotubes. J Appl Biomater Funct Mater. 2015;13(4): e301-e312. 
16. Zhang YB, Kanungo M, Ho AJ, et al. Functionalized carbon nanotubes for detecting viral proteins. Nano Lett. 2007;7(10):3086-3091.

17. Pan B, Cui D, Xu P, et al. Synthesis and characterization of polyamidoamine dendrimer-coated multi-walled carbon nanotubes and their application in gene delivery systems. Nanotechnology. 2009;20(12): 125101.

18. Zhang B, Chen Q, Tang H, et al. Characterization of and biomolecule immobilization on the biocompatible multi-walled carbon nanotubes generated by functionalization with polyamidoamine dendrimers. Colloids Surf B Biointerfaces. 2010;80(1):18-25.

19. Huang YP, Lin IJ, Chen CC, Hsu YC, Chang CC, Lee MJ. Delivery of small interfering RNAs in human cervical cancer cells by polyethylenimine-functionalized carbon nanotubes. Nanoscale Res Lett. 2013; 8(1):267.

20. Mashat A, Deng L, Altawashi A, Sougrat R, Wang G, Khashab NM. Zippered release from polymer-gated carbon nanotubes. J Mater Chem. 2012;22(23):11503-11508.

21. Masotti A, Miller MR, Celluzzi A, et al. Regulation of angiogenesis through the efficient delivery of microRNAs into endothelial cells using polyamine-coated carbon nanotubes. Nanomedicine. 2016;12(6): $1511-1522$.

22. Win KY, Feng SS. Effects of particle size and surface coating on cellular uptake of polymeric nanoparticles for oral delivery of anticancer drugs. Biomaterials. 2005;26(15):2713-2722.

23. Raffa V, Ciofani G, Nitodas S, et al. Can the properties of carbon nanotubes influence their internalization by living cells? Carbon $N Y$. 2008;46(12):1600-1610.

24. Yang K, Qin W, Tang H, et al. Polyamidoamine dendrimer-functionalized carbon nanotubes-mediated GFP gene transfection for HeLa cells: effects of different types of carbon nanotubes. J Biomed Mater Res A. 2011;99(2):231-239.

25. Liu D, Wang L, Wang Z, Cuschieri A. Different cellular response mechanisms contribute to the length-dependent cytotoxicity of multiwalled carbon nanotubes. Nanoscale Res Lett. 2012;7(1):361.

26. Martinelli A, Carru GA, D'Ilario L, et al. Wet adhesion of buckypaper produced from oxidized multiwalled carbon nanotubes on soft animal tissue. ACS Appl Mater Interfaces. 2013;5(10):4340-4349.

27. Loftus DJ, Leng T, Huie P, Fishman H, inventors; The United States of America as Represented by the Administrator of the National Aeronautics and Space Administration, assignee. Bucky paper as a support membrane in retinal cell transplantation. Patent US 7135172 B1. 2006 Nov 14.

28. Li Q, Liu C, Lin YH, Liu L, Jiang K, Fan S. Large-strain, multiform movements from designable electrothermal actuators based on large highly anisotropic carbon nanotube sheets. ACS Nano. 2015;9(1): $409-418$.

29. Adhikari BR, Govindhan M, Chen A. Carbon nanomaterials based electrochemical sensors/biosensors for the sensitive detection of pharmaceutical and biological compounds. Sensors (Basel). 2015;15(9): 22490-22508.

30. Zhao Q, Feng X, Mei S, Jin Z. Carbon-nanotube-assisted high loading and controlled release of polyoxometalates in biodegradable multilayer thin films. Nanotechnology. 2009;20(10):105101.

31. Correa-Duarte MA, Wagner N, Rojas-Chapana J, Morsczeck C, Thie M, Giersig M. Fabrication and biocompatibility of carbon nanotubebased 3D networks as scaffolds for cell seeding and growth. Nano Lett. 2004;4(11):2233-2236.
32. Deligianni DD. Multiwalled carbon nanotubes enhance human bone marrow mesenchymal stem cells' spreading but delay their proliferation in the direction of differentiation acceleration. Cell Adh Migr. 2014; $8(6): 558-562$.

33. Gottipati MK, Bekyarova E, Brenner M, Haddon RC, Parpura V. Changes in the morphology and proliferation of astrocytes induced by two modalities of chemically functionalized single-walled carbon nanotubes are differentially mediated by glial fibrillary acidic protein. Nano Lett. 2014;14(7):3720-3727.

34. Dinicola S, Masiello MG, Proietti S, et al. Multiwalled carbon nanotube buckypaper induces cell cycle arrest and apoptosis in human leukemia cell lines through modulation of AKT and MAPK signaling pathways. Toxicol In Vitro. 2015;29(7):1298-1308.

35. Weber J, Holman T, Eidenschink T, Chen JJ, inventors; Boston Scientific Scimed I, assignee. Using bucky paper as a therapeutic aid in medical applications. Patent US 8354120 B2. 2013 Jan 15.

36. Zhu N, Gao H, Xu Q, Lin Y, Su L, Mao L. Sensitive impedimetric DNA biosensor with poly(amido amine) dendrite covalently attached onto carbon nanotube electronic transducers as the tether for surface confinement of probe DNA. Biosens Bioelectron. 2010;25(6):1498-1503.

37. Li F, Peng J, Zheng Q, Guo X, Tang H, Yao S. Carbon nanotubepolyamidoamine dendrimer hybrid-modified electrodes for highly sensitive electrochemical detection of microRNA24. Anal Chem. 2015;87(9):4806-4813.

38. Lawson CL, Hanson RJ. Solving Least Square Problems. Englewood Cliffs, NJ: Prentice-Hall; 1974.

39. Tscharnuter WW. Mobility measurements by phase analysis. Appl Opt. 2001;40(24):3995-4003.

40. White B, Banerjee S, O'Brien S, Turro NJ, Herman IP. Zeta-potential measurements of surfactant-wrapped individual single-walled carbon nanotubes. J Phys Chem C. 2007;111(37):13684-13690.

41. Lane D, Prentki P, Chandler M. Use of gel retardation to analyze proteinnucleic acid interactions. Microbiol Rev. 1992;56(4):509-528.

42. Worle-Knirsch JM, Pulskamp K, Krug HF. Oops they did it again! Carbon nanotubes hoax scientists in viability assays. Nano Lett. 2006;6(6):1261-1268.

43. Masotti A, Moretti F, Mancini F, et al. Physicochemical and biological study of selected hydrophobic polyethylenimine-based polycationic liposomes and their complexes with DNA. Bioorg Med Chem. 2007; 15(3):1504-1515.

44. Pereira S, Lee J, Rubio N, et al. Cationic liposome-multi-walled carbon nanotubes hybrids for dual siPLK1 and doxorubicin delivery in vitro. Pharm Res. 2015;32(10):3293-3308.

45. Kobler C, Poulsen SS, Saber AT, et al. Time-dependent subcellular distribution and effects of carbon nanotubes in lungs of mice. PLoS One. 2015;10(1): $\mathrm{e} 0116481$.

46. Masotti A, Mossa G, Cametti C, et al. Comparison of different commercially available cationic liposome-DNA lipoplexes: parameters influencing toxicity and transfection efficiency. Colloids Surf B Biointerfaces. 2009;68(2):136-144.

47. Esposito C, Generosi J, Mossa G, Masotti A, Castellano AC. The analysis of serum effects on structure, size and toxicity of DDAB-DOPE and DC-Chol-DOPE lipoplexes contributes to explain their different transfection efficiency. Colloids Surf B Biointerfaces. 2006;53(2): 187-192. 


\section{Supplementary materials}

PAMAM

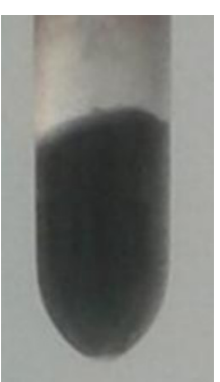

$10 \%$

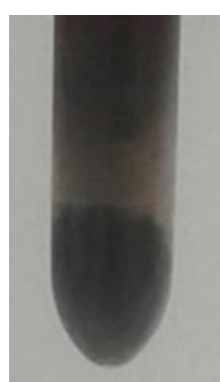

$20 \%$

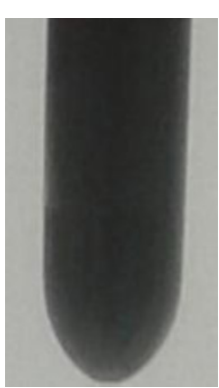

$40 \%$

Figure SI Preparation of PAM-CNTs starting from $10 \mathrm{mg}$ of pristine CNTs and different amounts of polymer solution (10\%, $20 \%$ and $40 \%$, w/w). Note: A stable suspension was obtained only using the $40 \%$ solution.

Abbreviations: CNTs, carbon nanotubes; PAMAM, polyamidoamine dendrimer.
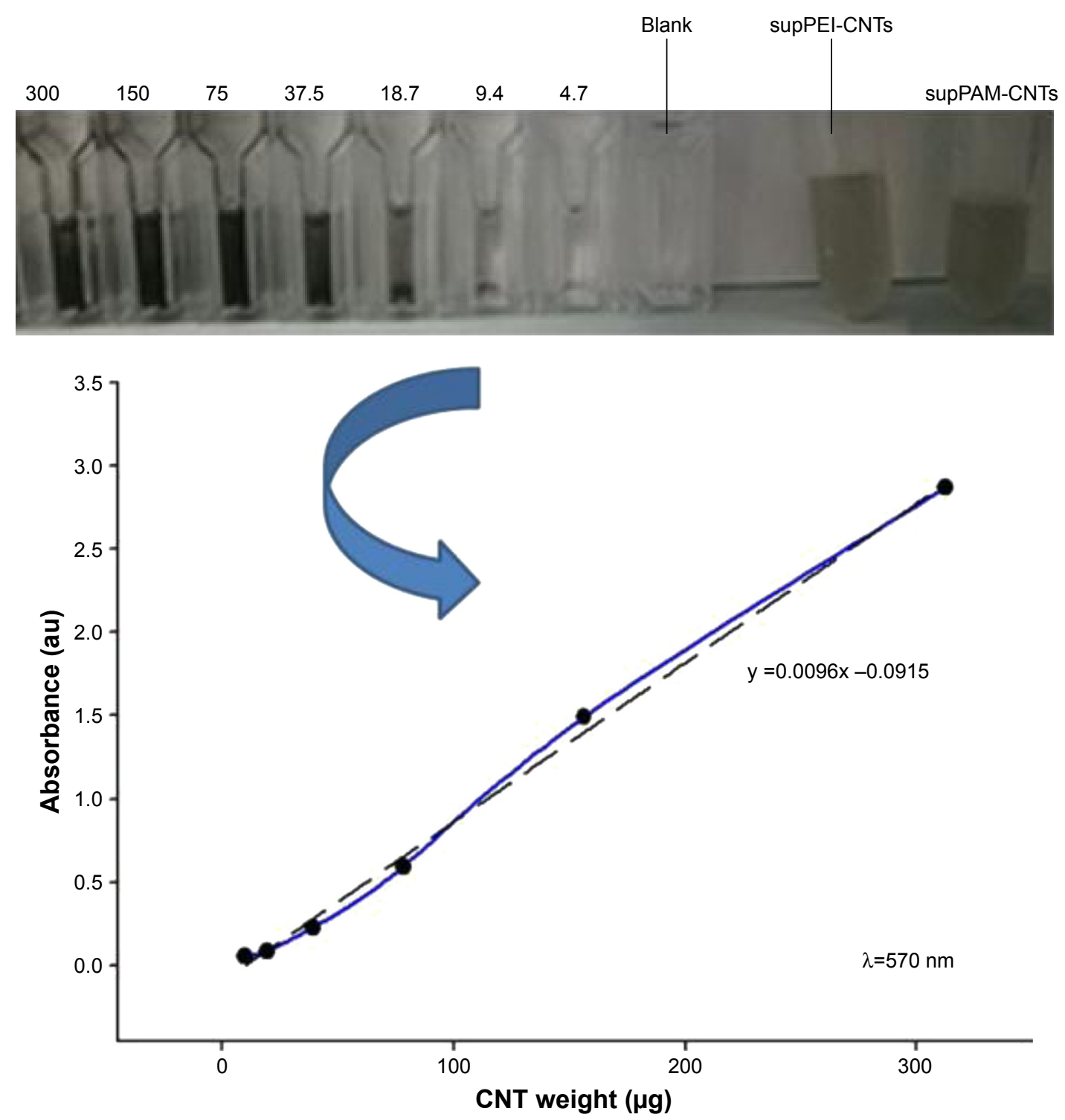

Figure S2 Calibration curve obtained by measuring absorbance $(570 \mathrm{~nm}$ ) of different dilutions (from 0 to $300 \mu \mathrm{g} / \mathrm{mL}$ ) of carbon nanotube dispersions. Note: Dotted line is the linear fit of the obtained data.

Abbreviations: CNTs, carbon nanotubes; PAMAM, polyamidoamine dendrimer; PEI, polyethyleneimine. 


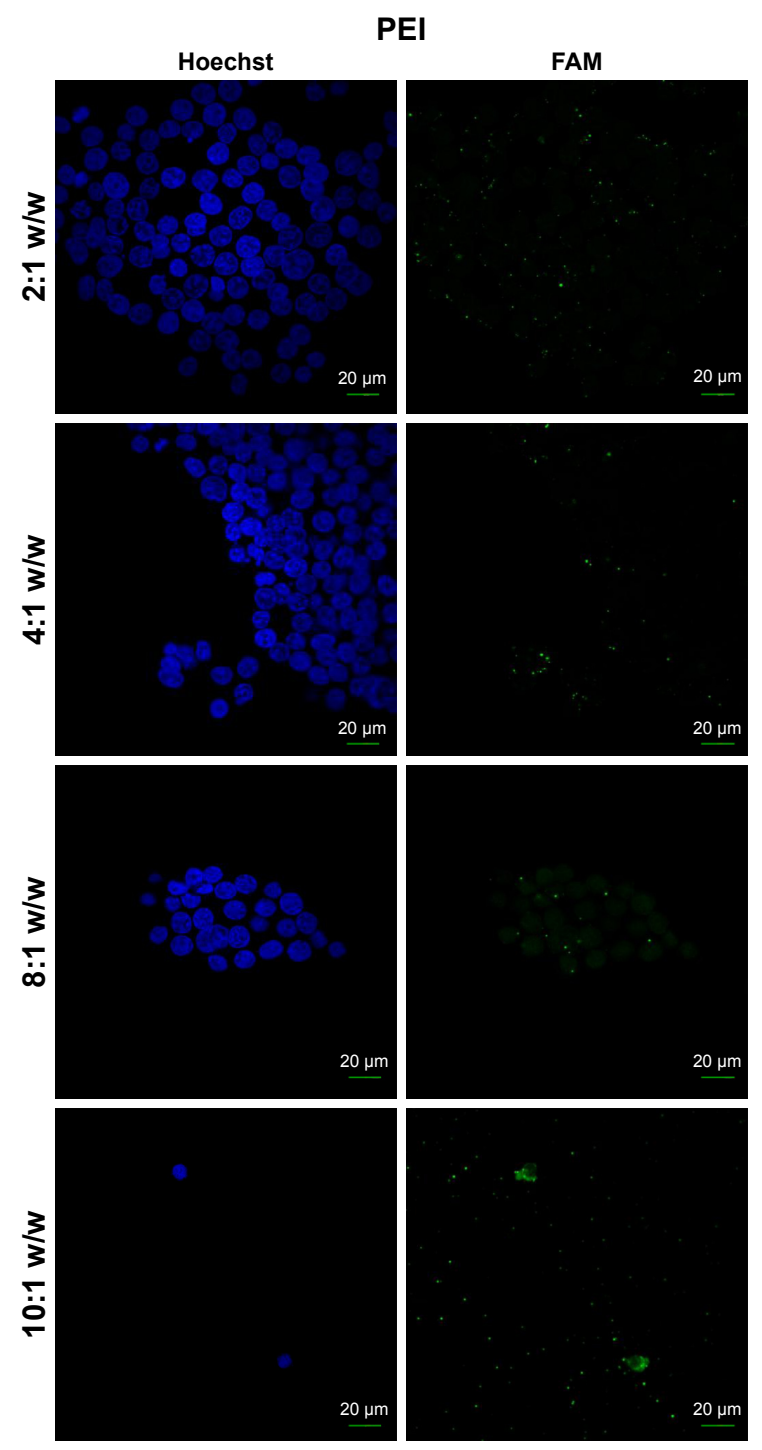

Figure S3 Fluorescence microscopy of HEK 293 cells treated with PEI/FAM-miR503 at different polymer to miRNA ratios $(\mathrm{w} / \mathrm{w})$ that indicate the best ratio to use in transfections.

Abbreviation: $\mathrm{PEI}$, polyethyleneimine.

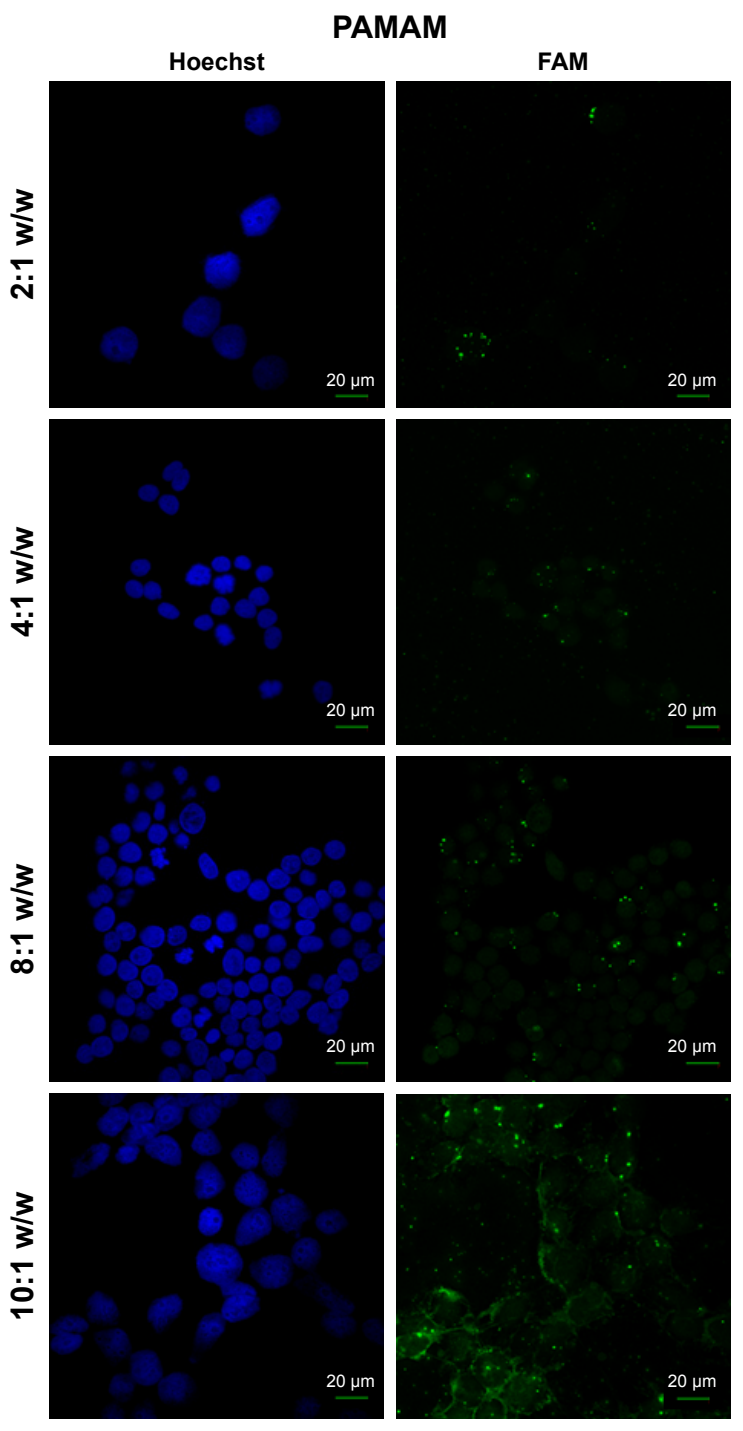

Figure S4 Fluorescence microscopy of HEK 293 cells treated with PAMAM/FAMmiR-503 at different polymer to miRNA ratios $(\mathrm{w} / \mathrm{w})$ that indicate the best ratio to use in transfections.

Abbreviation: PAMAM, polyamidoamine dendrimer.
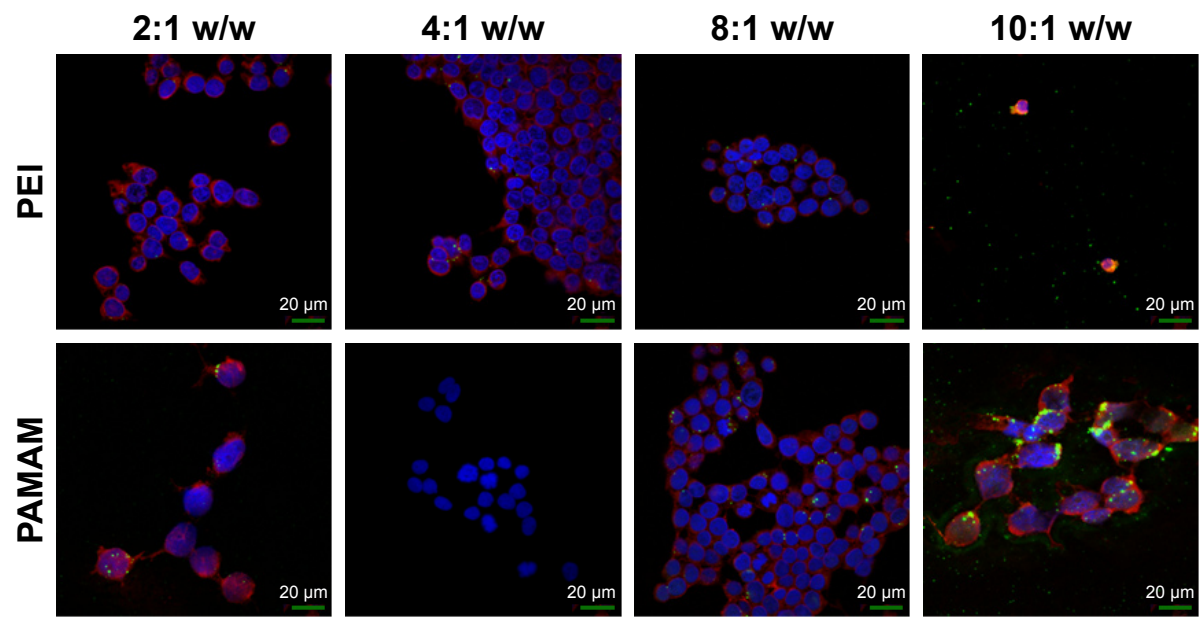

Figure S5 (Continued) 


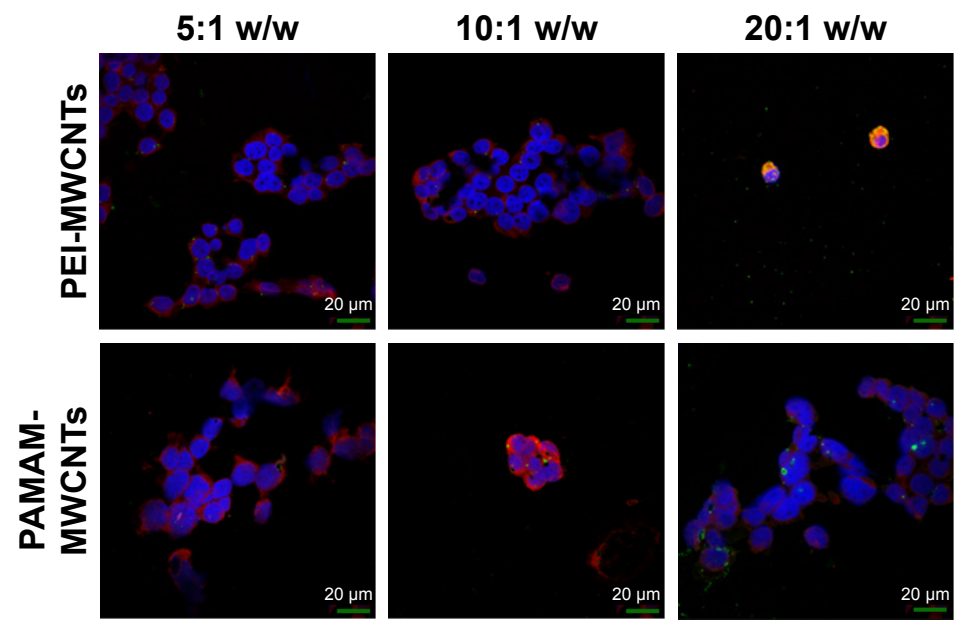

Figure S5 Fluorescence microscopy of HEK 293 cells treated with polymers compared to polymer-coated CNTs complexed with FAM-miR-503 at different weight ratios. Note: Magnification 60x.

Abbreviations: CNTs, carbon nanotubes; MWCNTs, multi-walled CNTs; PAMAM, polyamidoamine dendrimer; PEI, polyethyleneimine.
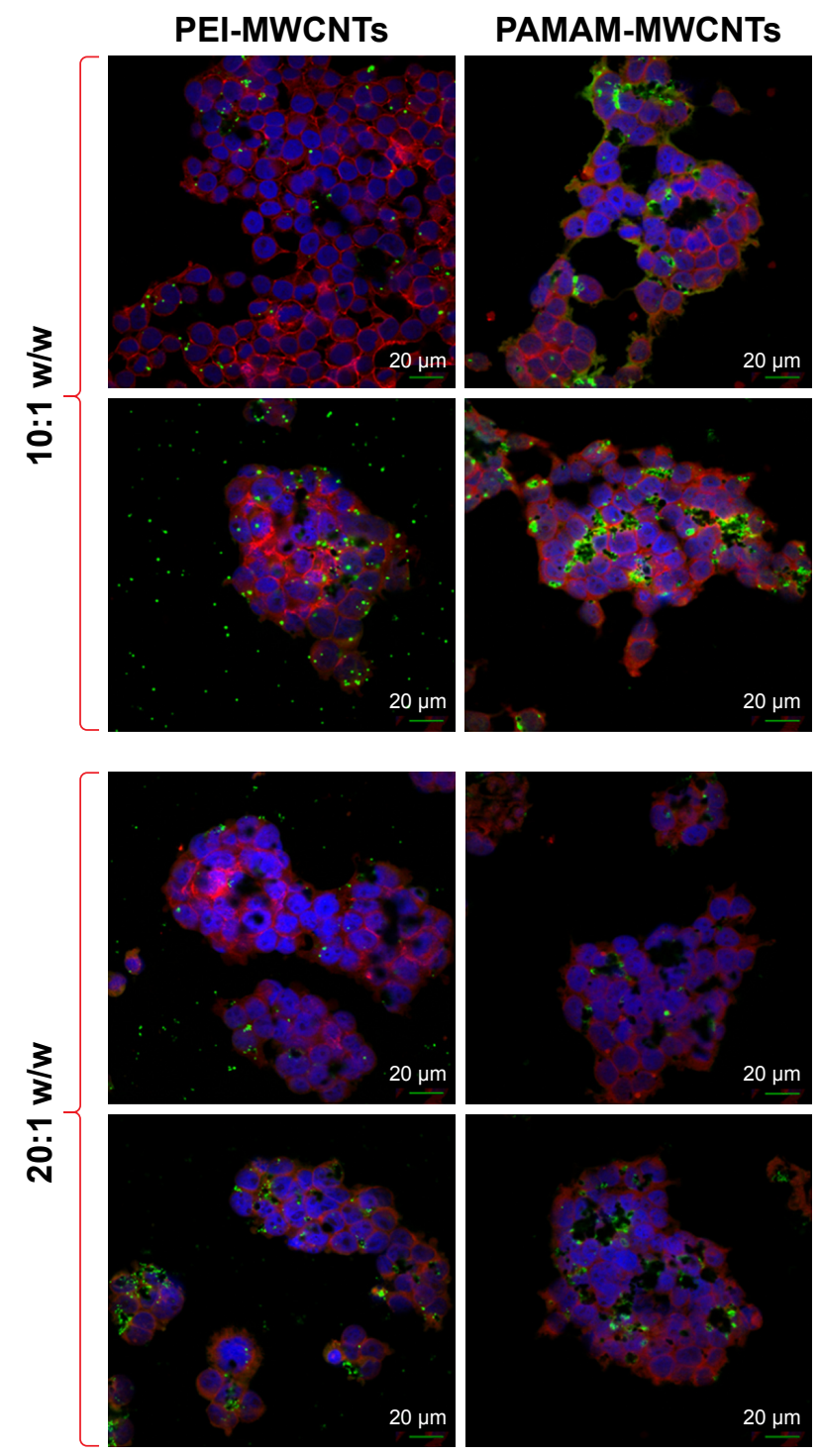

Figure S6 Fluorescence microscopy of HEK 293 cells treated with polymers compared to supernatants of polymer-coated CNTs. Notes: supernatants were complexed with FAM-miR-503 at 10:I and 20:I w/w ratio. Magnification 60x.

Abbreviations: CNTs, carbon nanotubes; MWCNTs, multi-walled CNTs; PAMAM, polyamidoamine dendrimer; PEl, polyethyleneimine. 


\begin{tabular}{lllll}
\hline Figure 6 & Area & Mean & IntDen & RawlntDen \\
\hline Blank & 48,168 & 4.651 & $\mathbf{2 2 4 , 0 5 3}$ & 224,053 \\
PEI 8:1 & 47,515 & 5.002 & $\mathbf{2 3 7 , 6 8 8}$ & 237,688 \\
PEI-CNTs 10:1 & 47,515 & 5.515 & $\mathbf{2 6 2 , 0 2 5}$ & 262,025 \\
PAMAM 8:1 & 47,736 & 5.958 & $\mathbf{2 8 4 , 4 2 9}$ & 284,429 \\
PAM-CNTs 20:1 & 47,515 & 6.404 & $\mathbf{3 0 4 , 2 7 8}$ & 304,278 \\
\hline
\end{tabular}

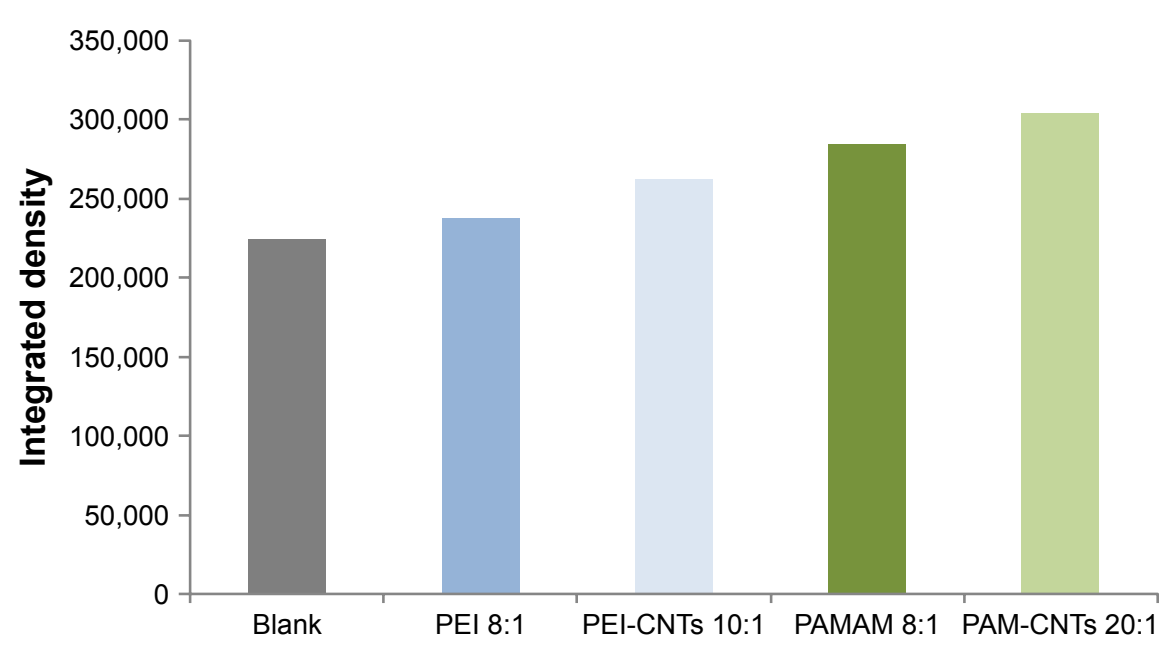

Figure S7 Fluorescence image densitometry of Figure 5 (main text).

Note: Absolute values presented in bold.

Abbreviations: CNTs, carbon nanotubes; PAMAM, polyamidoamine dendrimer; PEI, polyethyleneimine.

\section{Publish your work in this journal}

The International Journal of Nanomedicine is an international, peerreviewed journal focusing on the application of nanotechnology in diagnostics, therapeutics, and drug delivery systems throughout the biomedical field. This journal is indexed on PubMed Central, MedLine, CAS, SciSearch ${ }^{\circledR}$, Current Contents ${ }^{\circledR} /$ Clinical Medicine,
Journal Citation Reports/Science Edition, EMBase, Scopus and the Elsevier Bibliographic databases. The manuscript management system is completely online and includes a very quick and fair peer-review system, which is all easy to use. Visit http://www.dovepress.com/ testimonials.php to read real quotes from published authors. 